\title{
Redefinition of the self-bias voltage in a dielectrically shielded thin sheath RF discharge
}

Teck Seng Ho, Christine Charles, and Rod Boswell

Citation: Journal of Applied Physics 123, 193301 (2018); doi: 10.1063/1.5023076

View online: https://doi.org/10.1063/1.5023076

View Table of Contents: http://aip.scitation.org/toc/jap/123/19

Published by the American Institute of Physics

\section{Articles you may be interested in}

Laminar and turbulent flow modes of cold atmospheric pressure argon plasma jet Journal of Applied Physics 123, 193302 (2018); 10.1063/1.5012087

Enhancement of emission of InGaN/GaN multiple-quantum-well nanorods by coupling to Au-nanoparticle plasmons

Journal of Applied Physics 123, 193101 (2018); 10.1063/1.5022454

Performance modelling of plasma microthruster nozzles in vacuum

Journal of Applied Physics 123, 173301 (2018); 10.1063/1.5012765

Enhancement in magnetocaloric properties of $\mathrm{ErCrO}_{3}$ via A-site $\mathrm{Gd}$ substitution

Journal of Applied Physics 123, 193901 (2018); 10.1063/1.5022584

High quantum yield of the Egyptian blue family of infrared phosphors ( $\mathrm{MCuSi}_{4} \mathrm{O}_{10}, \mathrm{M}=\mathrm{Ca}, \mathrm{Sr}, \mathrm{Ba}$ )

Journal of Applied Physics 123, 193103 (2018); 10.1063/1.5019808

Tutorial: Determination of thermal boundary resistance by molecular dynamics simulations

Journal of Applied Physics 123, 191101 (2018); 10.1063/1.5027519

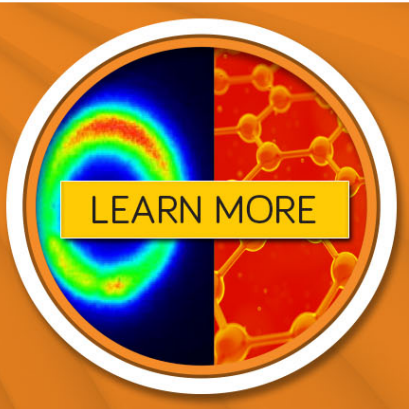




\title{
Redefinition of the self-bias voltage in a dielectrically shielded thin sheath RF discharge
}

\author{
Teck Seng Ho, Christine Charles, and Rod Boswell \\ Space Plasma, Power and Propulsion Laboratory, Research School of Physics and Engineering, \\ The Australian National University, Canberra, ACT 2601, Australia
}

(Received 21 January 2018; accepted 29 April 2018; published online 15 May 2018)

\begin{abstract}
In a geometrically asymmetric capacitively coupled discharge where the powered electrode is shielded from the plasma by a layer of dielectric material, the self-bias manifests as a nonuniform negative charging in the dielectric rather than on the blocking capacitor. In the thin sheath regime where the ion transit time across the powered sheath is on the order of or less than the Radiofrequency (RF) period, the plasma potential is observed to respond asymmetrically to extraneous impedances in the RF circuit. Consequently, the RF waveform on the plasma-facing surface of the dielectric is unknown, and the behaviour of the powered sheath is not easily predictable. Sheath circuit models become inadequate for describing this class of discharges, and a comprehensive fluid, electrical, and plasma numerical model is employed to accurately quantify this behaviour. The traditional definition of the self-bias voltage as the mean of the RF waveform is shown to be erroneous in this regime. Instead, using the maxima of the RF waveform provides a more rigorous definition given its correlation with the ion dynamics in the powered sheath. This is supported by a RF circuit model derived from the computational fluid dynamics and plasma simulations. Published by AIP Publishing. https://doi.org/10.1063/1.5023076
\end{abstract}

\section{INTRODUCTION}

Radiofrequency (RF) discharges are used in a wide variety of high technology industry applications, ranging from plasma processes such as reactive ion etching (RIE) and plasma enhanced chemical vapour deposition (PECVD) of thin films on substrate wafers for microelectronic fabrication to ion and electrothermal thrusters for electrically powered spacecraft propulsion. Ion bombardment is often a critical process in these applications. In plasma processing for example, there is a desire to understand and model ion bombardment to control etch profiles and etching rates, ${ }^{1}$ reduce sputtering and compositional or structural damage to the wafer surface, ${ }^{2}$ and address a range of other undesirable RIE-related effects. ${ }^{3}$ For spacecraft propulsion, hollow cathode thrusters ${ }^{4}$ rely on ion bombardment for heating and sustaining temperatures for thermionic emission, but sputtering and erosion of the insert and keeper electrode through the same process can severely limit the lifetime of the thruster. In the Pocket Rocket radiofrequency plasma electrothermal microthruster, ${ }^{5-7}$ ion bombardment plays a crucial role in the generation of secondary electrons for sustaining a gamma mode discharge.

In general, ion bombardment is a result of the acceleration of positive ions through a plasma sheath that spans a large potential drop, from the positive electric potential in the plasma bulk to the target surface that is at a lower electric potential. The target surface is often negatively biased, either by imposing a DC voltage or via the formation of a "selfbias." A self-bias can arise in a plasma system due to one or a combination of the following three scenarios: the secondary electron asymmetry effect (SEAE) resulting from the use of electrode materials with different affinities for secondary electron emission; ${ }^{8}$ the electrical asymmetry effect (EAE) due to using tailored waveforms at the powered electrode ${ }^{9,10}$ or from fluctuations in the plasma potential; ${ }^{11}$ or most commonly, using grounded and powered electrodes with a large area asymmetry ratio.

The self-bias phenomenon due to the geometrical area asymmetry of the grounded and powered electrodes has been extensively documented in experiment, ${ }^{12-16}$ simulation, ${ }^{17-19}$ and other literature. ${ }^{20}$ Essentially, when the ratio of the grounded and powered electrode areas is far from unity, the capacitance of the plasma sheath at the larger electrode is greater than that at the smaller electrode. In most plasma systems, the grounded electrode is often much larger than the powered electrode, resulting in a negative DC bias on the powered electrode that is maintained by a build-up of negative charge in a blocking capacitor, usually located in an external impedance matching circuit just before the powered electrode.

It is necessary to have an accurate characterisation of the self-bias behaviour in order to fully understand and control the ion bombardment process. However, performing measurements with invasive probes can be undesirable during operation or difficult due to geometry or size limitations in many plasma systems. For these situations, simulation is an especially useful tool for constructing a comprehensive model of the plasma system, which is capable of providing highly detailed data at unprecedented spatial and temporal resolutions.

\section{THEORY}

Capacitively coupled plasma (CCP) systems are often modelled as an electrical circuit consisting of two capacitors, representing the plasma sheaths at the grounded and powered electrodes, in series with a blocking capacitor and a RF 
source. In this picture, the circuit resembles a capacitive voltage divider, allowing for simple calculation of the root mean square (RMS) voltage drop across each capacitive component. However, this type of quasi-steady-state sheath circuit model ${ }^{21-25}$ is only valid in the regime where the ion transit time across the sheath is much greater than the RF period $\left(\tau_{\mathrm{i}} \gg \tau_{\mathrm{RF}}\right)$. In this regime, heavy ions do not respond to the time-varying high frequency RF electric field but rather to its time-average value. Sheath circuit models ${ }^{25-28}$ also exist for the opposite case of $\tau_{\mathrm{i}} \ll \tau_{\mathrm{RF}}$ in low frequency or high density (thin sheath) RF plasmas, where ion motion in the sheath is inertialess and responds rapidly to the oscillating RF electric field. In this regime, each sheath is modelled as a diode, current source, and capacitor in parallel, ${ }^{1,25}$ representing the electron current, ion current, and displacement current, respectively. Such a circuit has a very different voltage division outcome than the former case, and the calculation of the voltages across the sheaths as well as the blocking capacitor must take into account the electron and ion currents at each electrode during the positive and negative periods of the RF cycle.

The following is a summary of the derivation by Ref. 25 of the self-bias voltage $V_{\text {bias }}$ developed at the blocking capacitor in an ideal asymmetric RF discharge with a large grounded electrode and a small powered electrode with a time-varying voltage waveform $\Phi_{\mathrm{pwr}}(t)$.

Due to the blocking capacitor, no DC current can flow to either electrode. For this to be true, the plasma potential $\Phi_{\mathrm{p}}(t)$ must be positive relative to both electrodes; otherwise, more electrons reach the electrodes than ions due to their greater mobility. Additionally, for an asymmetric system, the smaller powered electrode must be negatively biased relative to the larger grounded electrode. This necessitates that $\Phi_{\mathrm{p}}(t)$ and the voltage across the powered sheath $\Phi_{\mathrm{p}}(t)$ $-\Phi_{\text {pwr }}(t)$ are alternately positive and clamped near zero. Consequently, the electron currents can only reach the electrodes when either sheath voltages are near zero and one or the other sheath alternately admits a pure ion current.

In the limit where $\tau_{\mathrm{i}} \ll \tau_{\mathrm{RF}}$, the plasma sheaths become resistive instead of capacitive as conduction currents dominate over displacement currents. Over one RF cycle, the total electron charge $Q_{\text {e,pwr }}$ collected by the powered electrode must equal the total ion charge collected

$$
Q_{\mathrm{e}, \mathrm{pwr}}=e u_{\mathrm{B}} n_{\mathrm{i}, \mathrm{pwr}} A_{\mathrm{pwr}} \tau_{\mathrm{RF}},
$$

where $e$ is the elementary charge, $u_{\mathrm{B}}$ is the local ion sound speed or Bohm velocity, $n_{\mathrm{i}, \mathrm{pwr}}$ is the plasma density at the inner edge of the powered sheath, $A_{\mathrm{pwr}}$ is the area of the powered electrode, and $\tau_{\mathrm{RF}}$ is the RF period.

Additionally, because the plasma must maintain quasineutrality during the positive period $\tau_{+}$of $\Phi_{\text {pwr }}(t)$, the total electron charge collected by the powered electrode must be equal to the total ion charge collected by both the powered and grounded electrodes during $\tau_{+}$

$$
Q_{\mathrm{e}, \mathrm{pwr}}=\left(e u_{\mathrm{B}} n_{\mathrm{i}, \mathrm{pwr}} A_{\mathrm{pwr}}+e u_{\mathrm{B}} n_{\mathrm{i}, \mathrm{gnd}} A_{\mathrm{gnd}}\right) \tau_{+},
$$

where $n_{\mathrm{i}, \text { gnd }}$ is the plasma density at the inner edge of the grounded sheath and $A_{\mathrm{pwr}}$ is the area of the grounded electrode.
Given that $\tau_{\mathrm{RF}}=\tau_{+}+\tau_{-}$, equating (1) and (2) gives

$$
\frac{\tau_{+}}{\tau_{-}}=\frac{u_{\mathrm{B}} n_{\mathrm{i}, \mathrm{pwr}} A_{\mathrm{pwr}}}{u_{\mathrm{B}} n_{\mathrm{i}, \mathrm{gnd}} A_{\mathrm{gnd}}}
$$

specifying that the ratio of the positive period to the negative period of $\Phi_{\mathrm{pwr}}(t)$ is proportional to the ratio of the ion current collected at each electrode. Finally, the solution for $\Phi_{\mathrm{pwr}}(t)$ to preserve $\tau_{+}$and $\tau_{-}$requires it to be displaced by the amount of

$$
V_{\text {bias }}=-V_{\mathrm{RF}} \sin \left(\frac{\pi}{2} \cdot \frac{u_{\mathrm{B}} n_{\mathrm{i}, \mathrm{gnd}} A_{\mathrm{gnd}}-u_{\mathrm{B}} n_{\mathrm{i}, \mathrm{pwr}} A_{\mathrm{pwr}}}{u_{\mathrm{B}} n_{\mathrm{i}, \mathrm{gnd}} A_{\mathrm{gnd}}+u_{\mathrm{B}} n_{\mathrm{i}, \mathrm{pwr}} A_{\mathrm{pwr}}}\right) .
$$

In a highly asymmetric system, assuming $A_{\text {gnd }} \gg A_{\text {pwr }}$ and $A_{\text {gnd }} / A_{\text {pwr }} \gg u_{\mathrm{B}} n_{\mathrm{i}, \mathrm{pwr}} / u_{\mathrm{B}} n_{\mathrm{i}, \text { gnd }}$ reduces (4) to the familiar $V_{\text {bias }}$ $=-V_{\mathrm{RF}}$.

This negative DC bias develops on the blocking capacitor such that the voltage waveform on the powered electrode is

$$
\Phi_{\mathrm{pwr}}(t)=-V_{\mathrm{RF}} \sin (\omega t)+V_{\text {bias }},
$$

where $V_{\mathrm{RF}}$ is the peak voltage of the sinusoidal RF waveform oscillating at the angular frequency $\omega=2 \pi f$. Since the DC $V_{\text {bias }}$ is negative and constant, $\Phi_{\mathrm{pwr}}(t)$ is displaced negatively relative to the supplied RF waveform by a fixed value, in the range $V_{\mathrm{RF}}-\left|V_{\text {bias }}\right| \geq \Phi_{\text {pwr }}(t) \geq-V_{\mathrm{RF}}-\left|V_{\text {bias }}\right|$.

The vital implication of this derivation for the $\tau_{\mathrm{i}} \ll \tau_{\mathrm{RF}}$ regime is that the $V_{\text {bias }}$ that develops on the blocking capacitor serves to preserve $\tau_{+}$and $\tau_{-}$, which results in a voltage division behaviour that is very different from the simpler capacitive sheath circuit model in the $\tau_{\mathrm{i}} \gg \tau_{\mathrm{RF}}$ regime. A numerical study by Ref. 28 uses the $\tau_{\mathrm{i}} \ll \tau_{\mathrm{RF}}$ regime sheath circuit model to calculate the ion energy distribution (IED) for varying capacitances and inductances in the external RF circuit while keeping the RF frequency and power fixed. The study finds that the IED is bimodal as expected, but the low energy peak remains constant and $V_{\text {bias }}$ is approximately constant despite changes in the impedances of the external RF circuit. Only the high energy peak is shifted when different capacitances and inductances are used but does not correlate with $V_{\text {bias }}$. This means that the positive peak of $\Phi_{\mathrm{pwr}}(t)$, corresponding to the minimum potential drop at the powered sheath, responsible for the low energy peak of the IED, does not depend on the size of the blocking capacitor or other impedances in the external RF circuit but is instead characteristic of the plasma parameters and the geometry of the plasma system. On the contrary, the negative peak of $\Phi_{\mathrm{pwr}}(t)$, corresponding to the maximum potential drop at the powered sheath, responsible for the high energy peak of the IED, is sensitive to the impedances of the external RF circuit. ${ }^{25}$ In an industrial RIE system for example, shifts in the ion bombardment energies might lead to undesirable process control. ${ }^{29}$ Even if the IED can be monitored with a retarding field energy analyser (RFEA), measurements taken in the $\tau_{\mathrm{i}} \ll \tau_{\mathrm{RF}}$ regime can be significantly affected by the RF modulated plasma sheath in front of the RFEA and thus not reflect the true IED. ${ }^{30}$

Up until now, $V_{\text {bias }}$ is defined to be the mean of the $\Phi_{\mathrm{pwr}}(t)$ waveform. However, since $\Phi_{\mathrm{pwr}}(t)$ responds 
asymmetrically to extraneous impedances in the RF circuit, using a more stable baseline like the maxima envelope $\Phi_{\mathrm{pwr}}^{+}$ $=V_{\mathrm{RF}}-\left|V_{\text {bias }}^{\prime}\right|$ instead can provide a more rigorous definition of the self-bias voltage (Sec. VD). This paper uses the Pocket Rocket geometrically asymmetric $13.56 \mathrm{MHz} \mathrm{RF}$ CCP discharge to demonstrate this phenomenon in the $\tau_{\mathrm{i}}$ $\sim 1.40 \tau_{\mathrm{RF}}$ regime, where the radial ion drift velocity $u_{r, \mathrm{i}}$ in the $1.2 \mathrm{~mm}$ thick collisional powered sheath varies by $\Delta u_{r, \mathrm{i}}$ $= \pm 27.1 \%$ within the RF cycle as a response to the oscillating RF electric field. ${ }^{31}$ In this regime, some assumptions of the $\tau_{\mathrm{i}} \ll \tau_{\mathrm{RF}}$ regime sheath circuit model are not explicitly valid, ${ }^{32,33}$ and a complete time-dependent treatment of ion inertial effects is required.

\section{APPARATUS}

Pocket Rocket (henceforth abbreviated as PR) is a radiofrequency plasma electrothermal microthruster currently under development by the Space Plasma, Power, and Propulsion (SP3) Laboratory at the Australian National University. ${ }^{7}$ At the core of PR (Fig. 1) is a hollow alumina $\left(\mathrm{Al}_{2} \mathrm{O}_{3}\right)$ dielectric refractory tube discharge chamber of dimensions $18 \mathrm{~mm}$ in length, $2.1 \mathrm{~mm}$ inner radius, and $1.0 \mathrm{~mm}$ wall thickness. A copper $(\mathrm{Cu})$ annular RF powered electrode is fitted coaxially around the outside middle section of the discharge chamber of dimensions $5 \mathrm{~mm}$ in length, $3.1 \mathrm{~mm}$ inner radius, and $8 \mathrm{~mm}$ outer radius. Macor discs enclose the powered electrode, electrically and thermally insulating it from the surrounding aluminium (Al) structure. The internal surface of the PR structure acts as the grounded electrode. It is much larger than the powered electrode; the area ratio of $A_{\text {gnd }} / A_{\text {pwr }} \approx 80$ makes PR a highly asymmetric system.

A $V_{\mathrm{RF}}=300 \mathrm{~V}$ (RMS RF voltage $\left.\bar{V}_{\mathrm{RF}}=212.1 \mathrm{~V}\right)$ sinusoidal waveform at $13.56 \mathrm{MHz}$ is applied to the powered electrode from a RF power supply through an impedance matching network (IMN). The IMN is a П-match consisting of two variable capacitors and an inductor, with a blocking capacitor connected after it in series. Inline digital voltage/ current $(\mathrm{V} / \mathrm{I})$ probes are used both before and after the IMN to measure pre- and post-match V/I values. The IMN has a quality factor of $Q \approx 10$ at the present RF voltage parameters. The voltage on the powered electrode is monitored by the post-match $\mathrm{V} / \mathrm{I}$ probe and manually adjusted at the RF power supply in the event of it drifting away from the set value. During operation, a negative DC bias voltage of $-1.10 \mathrm{~V}$ is registered at the blocking capacitor by the postmatch $\mathrm{V} / \mathrm{I}$ probe, which is a negligible value relative to $V_{\mathrm{RF}}$. This reading is separately confirmed with measurements using an analogue oscilloscope. This means that the dielectric discharge chamber wall acts as a blocking capacitor in its place, and most if not all of $V_{\text {bias }}$ manifests across the dielectric wall.

Argon (Ar) gas is flowed into PR through a $3 \mathrm{~mm}$ diameter inlet on one side of the plenum, which has dimensions of $12 \mathrm{~mm}$ in length and $20 \mathrm{~mm}$ internal radius. The static pressure of the gas is monitored using a capacitance manometer mounted to a port on the other side of the plenum. For a mass flow rate of $\dot{m}=2.97 \times 10^{-6} \mathrm{~kg} \mathrm{~s}^{-1}=100 \mathrm{SCCM}$ of Ar at $T=300 \mathrm{~K}$, the stagnation pressure in the plenum is a constant $p_{\text {st }}=1.365$ Torr. $\mathrm{PR}$ is mounted to a $20 \mathrm{~L}$ six-way cross vacuum chamber. When no gas is flowing, $\mathrm{PR}$ and the rest of the vacuum system achieve a base pressure of $\leq 1$ mTorr with a rotary vane pump connected to the vacuum chamber. Due to the small volume of the vacuum chamber and the limited pumping speed, the static pressure in the vacuum chamber rises to $p_{0}=0.349$ Torr with 100 SCCM of Ar. ${ }^{34}$

The Ar gas flowing through the discharge chamber under the powered electrode is ignited into a capacitively coupled plasma using $\sim 5 \mathrm{~W}$ of $\mathrm{RF}$ power. The oscillating RF electric fields accelerate an electron avalanche, and plasma breakdown occurs on an $\sim \mu$ s time scale. The discharge is initially symmetric, ${ }^{18}$ existing as an alpha mode sustained by bulk electrons, before transitioning to a gamma mode sustained by high energy secondary electrons, which are primarily generated by ion bombardment at the surface of the discharge chamber wall under the powered electrode

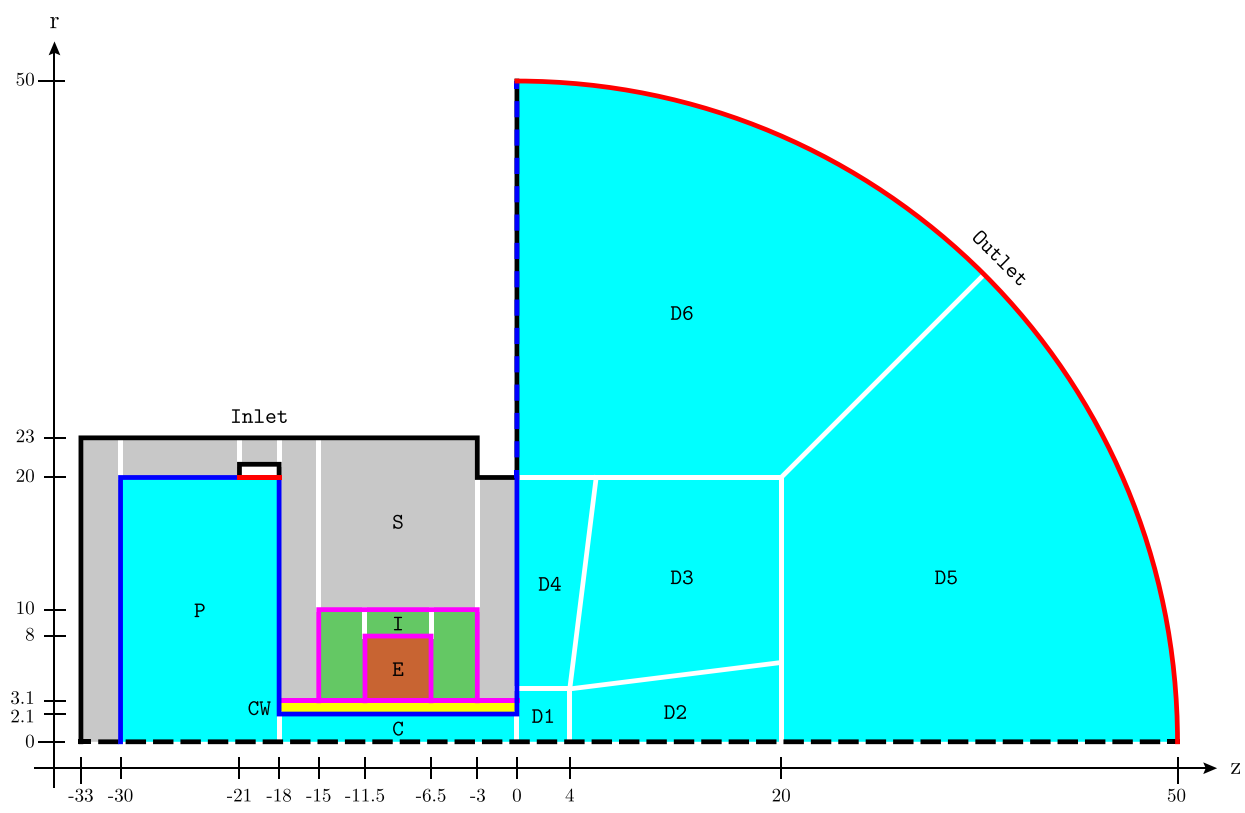

FIG. 1. 2D axisymmetric schematic of the PR simulation domain. Fluid regions (cyan): plenum $(\mathrm{P})$, discharge chamber (C), and downstream (D1 to D6). Solid regions: discharge chamber wall (CW, yellow), RF powered electrode (E, brown), insulation ( $I$, dark green), and grounded structure ( $S$, grey). Dimensions are given in units of $\mathrm{mm}$. Radial data presented in this paper are taken along $z=-9.9 \mathrm{~mm}$ where the plasma density peaks. 
after the formation of the self-bias. During plasma operation, the plasma extends upstream into the plenum and also a small distance out of the discharge chamber into the downstream region. ${ }^{35}$ Experimental measurements ${ }^{7,36-39}$ and simulation results $^{31,34,40,41}$ of the PR fluid, heating, and plasma parameters are published in these listed references.

\section{METHOD}

\section{A. Simulation mesh}

PR is modelled using the commercial computational fluid dynamics (CFD) and plasma multiphysics solver CFDACE +. A two dimensional axisymmetric mesh (Fig. 1) reproduces the physical device at actual scale in the simulation domain. A hemispherical downstream region with a radius of $50 \mathrm{~mm}$ represents the vacuum chamber. This geometry is selected as a hemispherical boundary is equidistant from the exit of the discharge chamber and isotropic, eliminating any directional bias and circulation effects that arise from boundaries at unequal distances, as well as computational anomalies caused by corners. Since the discharge chamber and the surrounding dielectric wall are the regions of primary interest for resolving fluid, electric, and plasma dynamics, the mesh density is the highest there with $0.1 \mathrm{~mm} \times 0.1 \mathrm{~mm}$ cells. The mesh density decreases smoothly outwards in the adjacent regions to save on computation time. Overall, the mesh density is higher than what is deemed sufficient by previous mesh independence studies. ${ }^{41}$

\section{B. Volume conditions}

Volume conditions for solid regions include parameters such as density, specific heat, thermal conductivity, electrical resistivity, and relative permittivity. For fluid regions, the density is given by the ideal gas law, and additional parameters include dynamic viscosity and mass diffusivity. All parameters loaded into the CFD-ACE + database are functions of temperature specific to the material or chemical species at $\sim 1$ Torr. Six species are considered in the CFDplasma simulation: $\operatorname{Ar}, \operatorname{Ar}\left(4 s_{m}\right), \operatorname{Ar}\left(4 s_{r}\right), \operatorname{Ar}(4 p), \mathrm{Ar}^{+}$, and $\mathrm{e}^{-}$, with 29 volumetric chemical reactions (Table I): elastic collision, 12 for electronic excitation and deexcitation, 2 for quenching, 4 for radiative decay, and 10 for direct, stepwise, and Penning ionisation. ${ }^{42,43}$ The discharge chamber is seeded with 0.999996 mass fraction of neutral Ar and $10^{-6}$ for each of the four other Ar species as the initial state, with the electron number density maintained by quasineutrality.

The chemistry model featured in the present CFDplasma simulations is a significant improvement to earlier works, ${ }^{41,44,45}$ where only four species ( $\mathrm{Ar}, \mathrm{Ar}^{*}, \mathrm{Ar}^{+}$, and $\mathrm{e}^{-}$) are considered, with only four reactions (elastic collision, electronic excitation, direct ionisation, and stepwise ionisation). Notably, no deexcitation, quenching, or radiative decay mechanisms are included previously. The inclusion of these loss pathways in the present simulations as part of a more complete reaction set results in a more accurate $\mathrm{Ar}^{+}$ density via the moderation of $\operatorname{Ar}\left(4 s_{m}\right), \operatorname{Ar}\left(4 s_{r}\right)$, and $\operatorname{Ar}(4 p)$ species, especially since deexcitation from $\operatorname{Ar}\left(4 \mathrm{~s}_{\mathrm{r}}\right)$ (reaction No. 9) and radiative decay from $\operatorname{Ar}(4 p)$ (reactions No.
17-19) occur at rates per unit volume that are on the same order of magnitude as direct ionisation. This in turn results in a more accurate calculation of the plasma potential and correspondingly the electric potential in the plasma sheaths and $V_{\text {bias }}$ in the dielectric discharge chamber wall.

\section{Boundary conditions}

The inlet boundary is set to admit a fixed $\dot{m}=100$ SCCM of $\operatorname{Ar}$ at $T=300 \mathrm{~K}$, while the outlet boundary is set to a fixed $p_{0}=0.349$ Torr to represent the measured pressure in the vacuum chamber, with a fixed backflow temperature $T=300 \mathrm{~K}$. A slip boundary condition with the appropriate tangential momentum and thermal accommodation coefficients is used for fluid-solid interfaces in the present CFDplasma simulations. As detailed previously, ${ }^{34}$ using the correct slip boundary condition is required for the simulation results to match experimental results for PR operating in the slip flow regime. A no-slip boundary condition overestimates static pressure and underestimate flow velocity, while an inviscid boundary condition results in the opposite effect and

TABLE I. Ar plasma chemical reaction set.

\begin{tabular}{|c|c|c|}
\hline No. & Reaction & Rate coefficient $^{43}$ \\
\hline \multicolumn{3}{|c|}{ Elastic collision } \\
\hline 1. & $\mathrm{Ar}+\mathrm{e}^{-} \rightarrow \mathrm{Ar}+\mathrm{e}^{-}$ & Collision cross section ${ }^{42}$ \\
\hline \multicolumn{3}{|c|}{ Electronic excitation/deexcitation } \\
\hline 2. & $\operatorname{Ar}+\mathrm{e}^{-} \rightarrow \operatorname{Ar}\left(4 \mathrm{~s}_{\mathrm{m}}\right)+\mathrm{e}^{-}$ & Collision cross section ${ }^{42}$ \\
\hline 3. & $\operatorname{Ar}+\mathrm{e}^{-} \rightarrow \operatorname{Ar}\left(4 \mathrm{~s}_{\mathrm{r}}\right)+\mathrm{e}^{-}$ & Collision cross section ${ }^{42}$ \\
\hline 4. & $\operatorname{Ar}+\mathrm{e}^{-} \rightarrow \operatorname{Ar}(4 \mathrm{p})+\mathrm{e}^{-}$ & Collision cross section ${ }^{42}$ \\
\hline 5. & $\operatorname{Ar}\left(4 s_{m}\right)+e^{-} \rightarrow \operatorname{Ar}+e^{-}$ & $4.3 \times 10^{-16} \cdot T_{\mathrm{e}}^{0.74}$ \\
\hline 6. & $\operatorname{Ar}\left(4 \mathrm{~s}_{\mathrm{m}}\right)+\mathrm{e}^{-} \rightarrow \operatorname{Ar}\left(4 \mathrm{~s}_{\mathrm{r}}\right)+\mathrm{e}^{-}$ & $3.7 \times 10^{-13}$ \\
\hline 7. & $\operatorname{Ar}\left(4 s_{m}\right)+e^{-} \rightarrow \operatorname{Ar}(4 p)+e^{-}$ & $8.9 \times 10^{-13} \cdot T_{\mathrm{e}}^{0.51} \cdot e^{-1.59 / T_{\mathrm{e}}}$ \\
\hline 8. & $\operatorname{Ar}\left(4 s_{\mathrm{r}}\right)+\mathrm{e}^{-} \rightarrow \operatorname{Ar}+\mathrm{e}^{-}$ & $4.3 \times 10^{-16} \cdot T_{\mathrm{e}}^{0.74}$ \\
\hline 9. & $\operatorname{Ar}\left(4 \mathrm{~s}_{\mathrm{r}}\right)+\mathrm{e}^{-} \rightarrow \operatorname{Ar}\left(4 \mathrm{~s}_{\mathrm{m}}\right)+\mathrm{e}^{-}$ & $9.1 \times 10^{-13}$ \\
\hline 10. & $\operatorname{Ar}\left(4 s_{r}\right)+e^{-} \rightarrow \operatorname{Ar}(4 p)+e^{-}$ & $8.9 \times 10^{-13} \cdot T_{\mathrm{e}}^{0.51} \cdot e^{-1.59 / T_{\mathrm{e}}}$ \\
\hline 11. & $\operatorname{Ar}(4 p)+e^{-} \rightarrow \operatorname{Ar}+e^{-}$ & $3.9 \times 10^{-16} \cdot T_{\mathrm{e}}^{0.71}$ \\
\hline 12. & $\operatorname{Ar}(4 \mathrm{p})+\mathrm{e}^{-} \rightarrow \operatorname{Ar}\left(4 \mathrm{~s}_{\mathrm{m}}\right)+\mathrm{e}^{-}$ & $3.0 \times 10^{-13} \cdot T_{\mathrm{e}}^{0.51}$ \\
\hline 13. & $\operatorname{Ar}(4 p)+\mathrm{e}^{-} \rightarrow \operatorname{Ar}\left(4 \mathrm{~s}_{\mathrm{r}}\right)+\mathrm{e}^{-}$ & $3.0 \times 10^{-13} \cdot T_{\mathrm{e}}^{0.51}$ \\
\hline \multicolumn{3}{|c|}{ Quenching } \\
\hline 14. & $2 \operatorname{Ar}\left(4 \mathrm{~s}_{\mathrm{m}}\right) \rightarrow 2 \mathrm{Ar}$ & $2.0 \times 10^{-13}$ \\
\hline 15. & $\operatorname{Ar}\left(4 \mathrm{~s}_{\mathrm{m}}\right)+\mathrm{Ar} \rightarrow 2 \mathrm{Ar}$ & $2.1 \times 10^{-21}$ \\
\hline \multicolumn{3}{|c|}{ Radiative decay } \\
\hline 16. & $\operatorname{Ar}\left(4 s_{\mathrm{r}}\right) \rightarrow \operatorname{Ar}+\hbar \omega$ & $1.0 \times 10^{5} \mathrm{~s}^{-1}$ \\
\hline 17. & $\operatorname{Ar}(4 p) \rightarrow \operatorname{Ar}+\hbar \omega$ & $3.2 \times 10^{7} \mathrm{~s}^{-1}$ \\
\hline 18. & $\operatorname{Ar}(4 \mathrm{p}) \rightarrow \operatorname{Ar}\left(4 \mathrm{~s}_{\mathrm{m}}\right)+\hbar \omega$ & $3.0 \times 10^{7} \mathrm{~s}^{-1}$ \\
\hline 19. & $\operatorname{Ar}(4 p) \rightarrow \operatorname{Ar}\left(4 s_{r}\right)+\hbar \omega$ & $3.0 \times 10^{7} \mathrm{~s}^{-1}$ \\
\hline \multicolumn{3}{|c|}{ Direct/stepwise/Penning ionisation } \\
\hline 20. & $\mathrm{Ar}+\mathrm{e}^{-} \rightarrow \mathrm{Ar}^{+}+2 \mathrm{e}^{-}$ & Collision cross section ${ }^{42}$ \\
\hline 21. & $\operatorname{Ar}\left(4 s_{m}\right)+\mathrm{e}^{-} \rightarrow \operatorname{Ar}^{+}+2 \mathrm{e}^{-}$ & $6.8 \times 10^{-15} \cdot T_{\mathrm{e}}^{0.67} \cdot e^{-4.20 / T_{\mathrm{e}}}$ \\
\hline 22. & $\operatorname{Ar}\left(4 s_{\mathrm{r}}\right)+\mathrm{e}^{-} \rightarrow \mathrm{Ar}^{+}+2 \mathrm{e}^{-}$ & $6.8 \times 10^{-15} \cdot T_{\mathrm{e}}^{0.67} \cdot e^{-4.20 / T_{\mathrm{e}}}$ \\
\hline 23. & $\operatorname{Ar}(4 \mathrm{p})+\mathrm{e}^{-} \rightarrow \mathrm{Ar}^{+}+2 \mathrm{e}^{-}$ & $1.8 \times 10^{-13} \cdot T_{\mathrm{e}}^{0.61} \cdot e^{-2.61 / T_{\mathrm{e}}}$ \\
\hline 24. & $2 \mathrm{Ar}\left(4 \mathrm{~s}_{\mathrm{m}}\right) \rightarrow \mathrm{Ar}^{+}+\mathrm{Ar}+\mathrm{e}^{-}$ & $6.4 \times 10^{-16}$ \\
\hline 25. & $2 \mathrm{Ar}\left(4 \mathrm{~s}_{\mathrm{r}}\right) \rightarrow \mathrm{Ar}^{+}+\mathrm{Ar}+\mathrm{e}^{-}$ & $6.4 \times 10^{-16}($ Same as No. 24$)$ \\
\hline 26. & $2 \mathrm{Ar}(4 \mathrm{p}) \rightarrow \mathrm{Ar}^{+}+\mathrm{Ar}+\mathrm{e}^{-}$ & $5.0 \times 10^{-16}$ \\
\hline 27. & $\operatorname{Ar}\left(4 s_{m}\right)+\operatorname{Ar}\left(4 s_{r}\right) \rightarrow \operatorname{Ar}^{+}+\operatorname{Ar}+e^{-}$ & $2.1 \times 10^{-15}$ \\
\hline 28. & $\operatorname{Ar}\left(4 s_{m}\right)+\operatorname{Ar}(4 p) \rightarrow \operatorname{Ar}^{+}+\operatorname{Ar}+e^{-}$ & $5.0 \times 10^{-16}($ Same as No. 26$)$ \\
\hline 29. & $\operatorname{Ar}\left(4 s_{r}\right)+\operatorname{Ar}(4 p) \rightarrow \operatorname{Ar}^{+}+\operatorname{Ar}+e^{-}$ & $5.0 \times 10^{-16}($ Same as No. 26) \\
\hline
\end{tabular}


gives unphysical results. The external boundaries of the simulation domain are allowed to freely radiate and exchange thermal energy with the surrounding air by convection, in an ambient $T=300 \mathrm{~K}$ atmospheric pressure environment that replicates the laboratory condition of the PR experiment. This allows for more accurate accounting of energy throughout all of the fluid and solid regions in the simulation domain, and also error checking as the temperature of the PR structure is not expected to rise by any significant amount during the time frame of the simulation. ${ }^{31}$

Additional boundary conditions are required to describe the electrical, chemistry, and plasma interactions between the fluid and solid surfaces. The structure surface (including the inlet boundary and the top left boundary of the downstream region) is defined to be electrically grounded, while the outlet boundary is defined as electrically floating. The discharge chamber wall surface is set to dielectric, while the boundaries of the powered electrode region are imposed with a $13.56 \mathrm{MHz}, V_{\mathrm{RF}}=300 \mathrm{~V}$ sinusoidal waveform. This is a valid assumption based on the post-match $\mathrm{V} / \mathrm{I}$ probe readings and since negligible bias voltage is registered on the blocking capacitor. Plasma-facing solid surfaces (i.e., fluid-solid interfaces and the top left wall of the downstream region) are defined with surface chemical reactions that transform incident $\operatorname{Ar}\left(4 s_{m}\right), \operatorname{Ar}\left(4 s_{r}\right), \operatorname{Ar}(4 p)$, and $\operatorname{Ar}^{+}$species to neutral Ar with a sticking coefficient of unity. These surfaces are set to have a secondary electron emission coefficient (SEEC) of $0.1{ }^{41}$ irrespective of the surface material. The effects of SEAE and EAE are excluded by using a constant SEEC and a single driving frequency of $13.56 \mathrm{MHz}$ on the powered electrode.

\section{Numerical method}

Modelling compressible flows in CFD-ACE+ requires the flow and heat transfer simulation modules. The addition of plasma to the simulation domain requires the electric, chemistry, and plasma modules. The solution methods for each of the modules have been defined previously. ${ }^{34,41,45}$ In addition to the base set of fluid and plasma equations, the presently employed technique also solves for electron stochastic heating and electron pressure, as well as for ion momentum, mobility, ohmic heating, and surface heating from ion bombardment.

The CFD-plasma simulations are solved in a transient manner in order to capture the time-dependent plasma dynamics within the RF cycle, unlike previous CFD cold gas simulations ${ }^{34}$ which have steady state solutions. Two distinct solver time-steps are used in the present CFD-plasma simulations: $\Delta \tau_{\mathrm{f}}=1.0 \mu \mathrm{s}$ for fluid dynamics and $\Delta \tau_{\mathrm{p}}=1.229 \mathrm{~ns}$ for plasma dynamics, equivalent to $1 / 60$ of the period $\tau_{\mathrm{RF}}$ $=73.7 \mathrm{~ns}$ of a $13.56 \mathrm{MHz} \mathrm{RF}$ cycle. 60 time-steps per RF cycle are chosen since $\sim 1 \mathrm{~ns}$ is the recommended time-step resolution for resolving plasma dynamics and performs best with the $V_{\mathrm{RF}}=300 \mathrm{~V}$ waveform used presently without being too excessive. $\Delta \tau_{\mathrm{f}}$ and $\Delta \tau_{\mathrm{p}}$ are used for solving their corresponding equations independently, without any intermediate time-stepping, and do not have to be synchronised. Some solvers like COMSOL are limited to using a single time-step for solving the entire system of equations for both electron and heavy particle dynamics. ${ }^{46}$ However, doing this or setting $\Delta \tau_{\mathrm{f}}$ too small (e.g., $1 \mu \mathrm{s}$ ) causes the fluid to either be frozen in time or the solution to be unstable and divergent, while setting $\Delta \tau_{\mathrm{f}}$ too large (e.g., $1 \mathrm{~ms}$ or even $1 \mathrm{~s}$ ) causes large unphysical fluctuations and divergent behaviour. For best results, $\Delta \tau_{\mathrm{f}}$ has to be set to a value commensurate with the dynamics of the problem.

The main feature of this dual time-step technique is that different simulation modules run at different rates. Two distinct iterative cycles are used in the CFD-plasma simulations. The first is the "fluid iteration" $i_{\mathrm{f}}$, which involves the flow, heat transfer, and chemistry modules, controlling parameters such as flow velocity and pressure, and are resolved at $\Delta \tau_{\mathrm{f}}$. The second is the "plasma iteration" $i_{\mathrm{p}}$, which involves the electric and plasma modules, controlling parameters such as electron number density, electron temperature, and electric potential, and are resolved at $\Delta \tau_{\mathrm{p}}$. Temperature, chemical reactions, and the mass fractions of heavy species are also controlled by $i_{\mathrm{f}}$, but these parameters are solved intelligently on a time-step that bridges $\Delta \tau_{\mathrm{f}}$ and $\Delta \tau_{\mathrm{p}}$. The value of $i_{\mathrm{f}}$ is selected empirically, scaling based on the complexity of the flow behaviour and the degree of interaction between the fluid and the plasma, while $i_{\mathrm{p}}$ scales inversely with pressure.

The present technique uses $i_{\mathrm{f}}=10$ for slip regime supersonic flow with significant plasma heating and $i_{\mathrm{p}}=5$ for an $\sim 1$ Torr simulation domain. This means that the electric and plasma modules cycle for five iterations among themselves, before passing the results at the end of the fifth plasma iteration to the flow, heat transfer, and chemistry modules. The flow, heat transfer, and chemistry modules then cycle once and then return their results to the electric and plasma modules. The process then repeats for a total of ten times, whereupon the final result at the end of the tenth fluid iteration constitutes the solution of the first simulation time-step. During each time-step, the electric and plasma parameters advance by $\Delta \tau_{\mathrm{p}}$, while the fluid parameters advance by $\Delta \tau_{\mathrm{f}}$. At the end of 60 time-steps, the electric and plasma parameters have advanced by $\tau_{\mathrm{RF}}$ or exactly one RF cycle, while the fluid parameters have advanced by $60 \mu \mathrm{s}$. Since $\Delta \tau_{\mathrm{f}}$ is not synchronised to $\Delta \tau_{\mathrm{p}}$, the fluid parameters must be averaged over the RF cycle for valid interpretation.

The present technique first runs the simulation for 100 RF cycles (6000 time-steps) from static initial conditions but with $i_{\mathrm{f}}=1$ to obtain a quick but inaccurate "seed solution." This seed solution is used as the initial conditions to start the "convergence run," which goes for 1000 RF cycles (60000 time-steps). As this process takes about a fortnight on a second generation Intel Core processor clocked at $4.5 \mathrm{GHz}$, the convergence run is broken into ten stages to mitigate data loss in the event of failure. The solution at the end of the convergence run is used as the initial conditions to start the "final run" of $20 \mathrm{RF}$ cycles (1200 time-steps). The results from first half of the final run are not used as anomalies have been known to appear when using previous solutions as initial conditions, but these anomalies tend to disappear quickly within a few cycles and the solution returns to equilibrium shortly thereafter. Hence, the latter $10 \mathrm{RF}$ cycles (600 time-steps) 
constitute the "final solution" from which the results presented in this paper are obtained.

It is critical that the simulation settings (e.g., $\Delta \tau_{\mathrm{f}}, \Delta \tau_{\mathrm{p}}, i_{\mathrm{f}}$, $i_{\mathrm{p}}$, and the number of time-steps per RF cycle) are not changed after committing to the convergence run process. Previous CFD-plasma simulations of $\mathrm{PR}^{41,44,45}$ have used only 20 time-steps per RF cycle during the convergence run to reduce computation time. However, subsequent tests have shown that the solution at the end of the convergence run is of lower quality than using the present settings, with some significant differences in the results. Although 60 time-steps per cycle are used for the final few RF cycles in those simulations, the time is insufficient for capturing any further evolution of the solution and may thus produce erroneous results. This issue has been resolved in the present simulation technique.

\section{RESULTS AND DISCUSSION}

\section{A. Electric potential overview}

Figure 2 shows a spatiotemporal overview of the electric potential $\Phi(t)$ in the different regions within PR, radially along $z=-9.9$ (blue lines), from the peak plasma density position at $(z, r)=(-9.9,0)$, through the discharge chamber (cyan), discharge chamber wall (yellow, $2.1<r<3.1$ ), powered electrode (brown, $3.1<r<8$ ), insulation (dark green, $8<r<10$ ), and the structure (grey) beyond. The $10 \mathrm{RF}$ cycles in the final solution are averaged to give a single characteristic RF cycle with 60 time-steps. There are 60 lines in the profile representing $\Phi(t)$ evaluated at each time-step of the characteristic RF cycle, while the single solid line in the middle of the profile represents the mean $\Phi$ over the period of the characteristic RF cycle.

The structure (grey) is defined to be electrically grounded, and therefore, $\Phi(t)$ is always zero in the electrically conductive $\mathrm{Al}$ region. In the powered electrode (brown), the imposed sinusoidal voltage waveform $\Phi_{\mathrm{pwr}}(t)$ causes the whole electrically conductive $\mathrm{Cu}$ region to

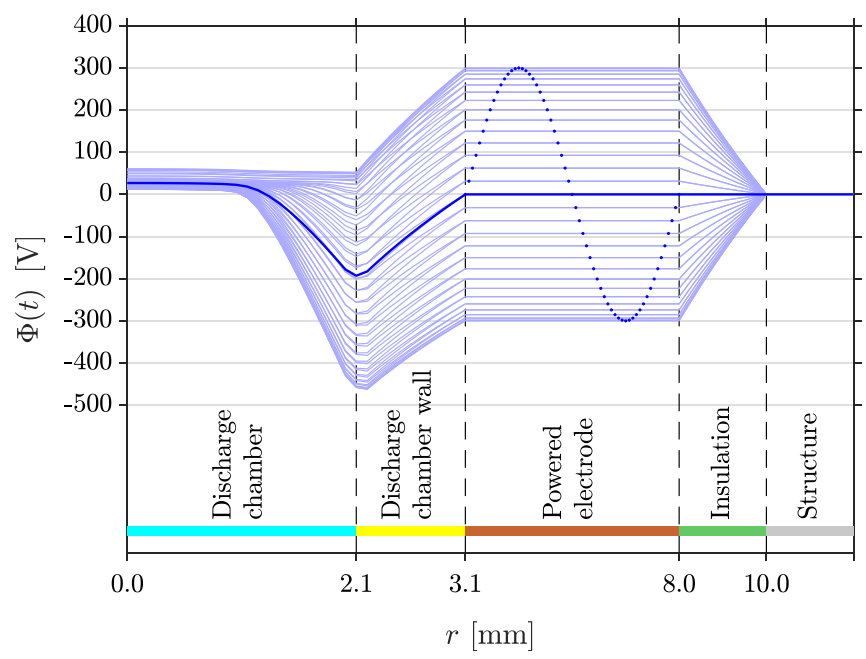

FIG. 2. Spatially and temporally resolved electric potential $\Phi(t)$ profile through different regions of PR plotted along the radial axis on $z=-9.9$ (blue lines). The solid line denotes the mean $\Phi$, while the dotted line illustrates the oscillation of $\Phi_{\mathrm{pwr}}(t)$ in the powered electrode over one RF cycle. oscillate between $\pm 300 \mathrm{~V}$. A layer of dielectric insulation (dark green) separates the powered electrode from the grounded structure. An electric displacement field is created in the dielectric Macor region that bridges $\Phi(t)$ between the powered electrode and the grounded structure. In these solid regions, the mean electric potential $\Phi$ over one RF cycle (solid line) is zero.

The discharge chamber wall (yellow) however exhibits a rather different behaviour. The displacement field in the dielectric $\mathrm{Al}_{2} \mathrm{O}_{3}$ region, as evidenced by the gradient $\nabla_{r} \Phi(t)$, appears to be approximately constant over the whole RF cycle, with the electric potential at the plasma-facing surface of the wall $\Phi_{\text {wall }}(t)$ being biased negatively relative to $\Phi_{\mathrm{pwr}}(t)$ on the powered electrode side. This is the manifestation of the self-bias due to the geometrical asymmetry of the plasma system. At the coordinates $(z, r)=(-9.9,2.1)$, the mean electric potential is $\Phi_{\text {wall }}=-193 \mathrm{~V}$, while the maximum and minimum values are $\Phi_{\text {wall }}^{+}=53 \mathrm{~V}$ and $\Phi_{\text {wall }}^{-}=-458 \mathrm{~V}$, respectively. This gives a peak-to-peak range of $\left|\Phi_{\text {wall }}^{+}-\Phi_{\text {wall }}^{-}\right|=511 \mathrm{~V}$, equivalent to $85 \%$ of the supplied $600 \mathrm{~V}$.

In the discharge chamber (cyan), the plasma potential $\Phi_{\mathrm{p}}(t)$ peaks at the same location as the plasma density peak. At the coordinates $(z, r)=(-9.9,0)$, the mean plasma potential is $\Phi_{\mathrm{p}}=-27.0 \mathrm{~V}$, while $\Phi_{\mathrm{p}}^{+}=61 \mathrm{~V}$ and $\Phi_{\mathrm{p}}^{-}=12.0 \mathrm{~V}$, indicating that $\Phi_{\mathrm{p}}(t)$ oscillates by $\left|\Phi_{\mathrm{p}}^{+}-\Phi_{\mathrm{p}}^{-}\right|=49 \mathrm{~V}$ within the RF cycle. $\Phi_{\mathrm{p}}(t)$ is positive and higher than $\Phi_{\mathrm{pwr}}(t)$ during $\tau_{+}$and clamped near zero during $\tau_{-}$. Conversely, the potential difference across the powered sheath $\Phi_{\mathrm{p}}(t)-\Phi_{\mathrm{pwr}}(t)$ is clamped near zero during $\tau_{+}$and greatly positive during $\tau_{-}$. On the other hand, the potential difference across the grounded sheath along the plasma-facing surfaces of the structure (not shown in Fig. 2) is much smaller in this highly asymmetric plasma system. These results are in agreement with the theoretical analysis outlined in Sec. II.

\section{B. Dielectric wall and sheath capacitances}

To study the effects of varying the dielectric wall capacitance on the self-bias, two additional CFD-plasma simulations of PR are run with $0.5 \mathrm{~mm}$ and $1.5 \mathrm{~mm}$ thick discharge chamber walls. To distinguish between the three simulations, the default configuration with a $1.0 \mathrm{~mm}$ thick wall shall be designated as PR-10, while the two new configurations shall be designated as PR-05 and PR-15. In these simulations, only the external radius of the discharge chamber wall is modified, so as to preserve $A_{\mathrm{gnd}} / A_{\mathrm{pwr}}$.

The capacitance of a cylindrical dielectric capacitor is given by

$$
C=\frac{2 \pi \varepsilon_{0} \varepsilon_{\mathrm{r}} L}{\ln \left(\frac{R}{r}\right)},
$$

where $\varepsilon_{0}=8.854 \times 10^{12} \mathrm{~F} \mathrm{~m}^{-1}$ is the vacuum permittivity, $\varepsilon_{\mathrm{r}}$ is the relative permittivity of the dielectric, $L$ is the length of the cylinder, and $R$ and $r$ are its outer and inner radii, respectively. Hence, the capacitance of the section of the $\varepsilon_{\mathrm{r}}=9.6$ $\mathrm{Al}_{2} \mathrm{O}_{3}$ wall immediately in front of the $L=5 \mathrm{~mm}$ powered electrode is $C_{\mathrm{wall}}=12.5 \mathrm{pF}, 6.9 \mathrm{pF}$, and $5.0 \mathrm{pF}$ for PR-05, 
PR-10, and PR-15, respectively, which equates to an impedance of $\left|Z_{\text {wall }}\right|=\left|-1 / \omega C_{\text {wall }}\right|=0.94 \mathrm{k} \Omega, 1.7 \mathrm{k} \Omega$, and $2.4 \mathrm{k} \Omega$, respectively. For comparison, the capacitance of the blocking capacitor used in the PR RF circuit is $C_{\text {block }}=100 \mathrm{pF}$.

Equivalent capacitance values can also be calculated for the powered sheath using (6) and $\varepsilon_{\mathrm{r}}=1$ to give $C_{\mathrm{s}, \mathrm{pwr}}^{\prime} \approx 3.2 \mathrm{pF}$. This calculation uses the outer and inner radii of the powered sheath in $\mathrm{PR}-10^{31}$ with $R=2.1 \mathrm{~mm}$ and $r=0.9 \mathrm{~mm}$ but remains approximately valid in $\mathrm{PR}-05$ and PR-15 where the sheath thickness is only very slightly different. Due to the larger size of the grounded sheath, it may be approximated by a parallel plate capacitor with an area equivalent to the internal grounded surface of the structure of the plenum plus the surface of the end wall facing the downstream region. The width of the grounded sheath at the front wall of the plenum is approximately $d=1.94 \mathrm{~mm}$, so $C_{\mathrm{s} \text {,gnd }}^{\prime}=\varepsilon_{0} A_{\text {gnd }} / d \approx 229 \mathrm{pF}$, with a slight variation of $\lesssim 1 \mathrm{pF}$ between the three configurations due to different inner radii of the rear and end walls as a result of the modified external radius of the discharge chamber wall. The collisional sheath capacitance equation $C_{\mathrm{s} \text { sym }} \approx 0.76 \cdot \varepsilon_{0} A_{\mathrm{sym}} / d$ is not able to be used for $\mathrm{PR}$ as it is only valid for symmetric plasma systems. $^{22,24}$

The $C_{\mathrm{s}, \mathrm{pwr}}^{\prime}$ and $C_{\mathrm{s} \text {,gnd }}^{\prime}$ values quoted above are not necessarily accurate in reality, especially in the $\tau_{\mathrm{i}} \ll \tau_{\mathrm{RF}}$ regime where the plasma sheaths become resistive instead of capacitive. Instead, it is more appropriate to quantify their impedances, which in the $\tau_{\mathrm{i}} \sim \tau_{\mathrm{RF}}$ regime is some combination of resistance $R$ and capacitive reactance $X_{C}=-1 / \omega C$. Since $R$ $\propto d / A$ uses the same geometrical parameters as $X_{C} \propto d / A$, the effective impedance is therefore $|Z|=\sqrt{R^{2}+X_{C}^{2}}$ $\propto\left|X_{C}\right|$. Hence, the powered and grounded sheath impedances are $\left|Z_{\mathrm{s}, \mathrm{pwr}}^{\prime}\right| \approx z \cdot 3.7 \mathrm{k} \Omega$ and $\left|Z_{\mathrm{s}, \text { gnd }}^{\prime}\right| \approx z \cdot 51 \Omega$, respectively, where $z$ is an unknown proportionality constant.

Figures 3 and 4 give a closer look at the electric potential $\Phi^{+}$and $\Phi^{-}$during the positive $\left(\tau_{+}\right)$and negative $\left(\tau_{-}\right)$

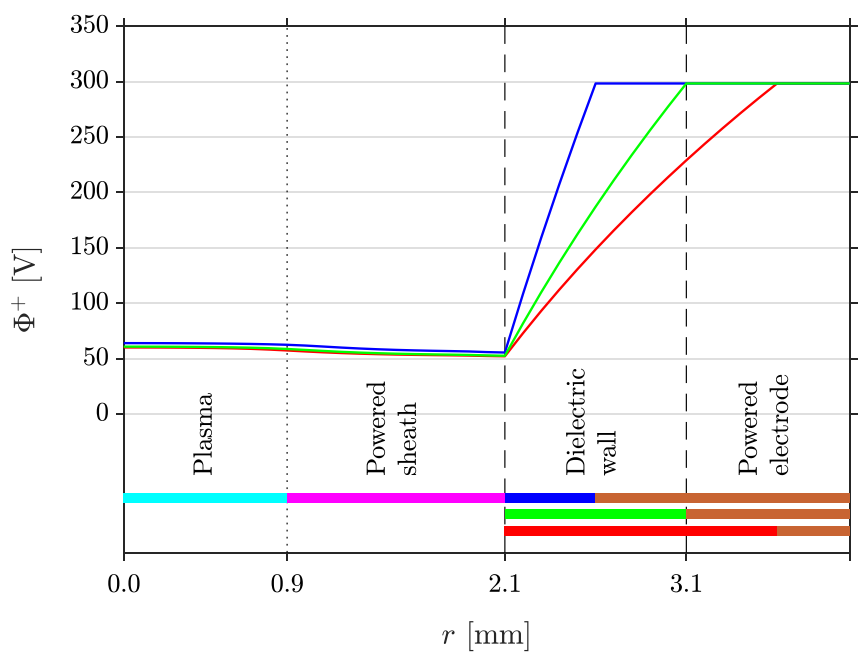

FIG. 3. Electric potential $\Phi^{+}$during the positive peak of the RF cycle through different regions of PR-05 (blue solid line), PR-10 (green solid line), and PR-15 (red solid line). The plasma potential $\Phi_{\mathrm{p}}^{+}$profile remains largely similar across the three configurations. The blue, green, and red bars on the bottom of the plot show the thickness of the dielectric wall in the respective configurations.

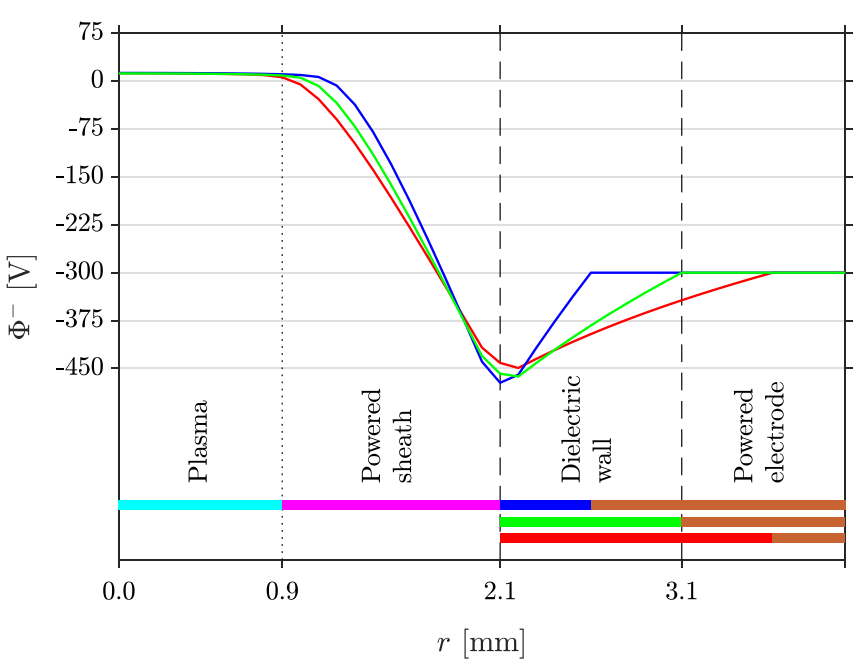

FIG. 4. Electric potential $\Phi^{-}$during the negative peak of the RF cycle through different regions of PR-05 (blue solid line), PR-10 (green solid line), and PR-15 (red solid line). The plasma potential $\Phi_{\mathrm{p}}^{-}$profile remains largely similar across the three configurations, but the gradient $\nabla_{r} \Phi_{\mathrm{p}}^{-}$in the powered sheath and the electric potential at the plasma-facing surface of the discharge chamber wall $\Phi_{\text {wall }}^{-}$are slightly different due to the different dielectric wall capacitances $C_{\text {wall }}$.

peaks of the RF cycle, respectively, averaged over the $10 \mathrm{RF}$ cycles in the final results. Different coloured lines represent $\Phi^{+}$and $\Phi^{-}$from PR- 05 (red solid line), PR-10 (green solid line), and PR-15 (blue solid line). The horizontal axes are labelled according to the plasma and RF circuit features instead of the physical regions of $\mathrm{PR}$, tracing a path from the plasma bulk (cyan), through the powered sheath (magenta), dielectric wall (colour coded according to each configuration), to the powered electrode (brown). Note that the vertical axes are at different scales in the two figures. Despite significant changes in the dielectric wall capacitance $C_{\text {wall }}$, the plasma potential $\Phi_{\mathrm{p}}^{+}$and $\Phi_{\mathrm{p}}^{-}$profiles remain largely similar across the three configurations. Figures 3 and 4 also demonstrate the alternately positive $\left(\Phi_{\mathrm{p}}^{+}\right.$and $\left.\Phi_{\mathrm{p}}^{-}-\Phi_{\text {wall }}^{-}\right)$and clamping $\left(\Phi_{\mathrm{p}}^{-}\right.$and $\left.\Phi_{\mathrm{p}}^{+}-\Phi_{\text {wall }}^{+}\right)$behaviour of the plasma potential and the potential difference across the powered sheath, as well as the $\Phi_{\mathrm{p}}(t)>\Phi_{\text {wall }}(t)$ behaviour mentioned earlier. The maxima, minima, peak-to-peak, and mean values of $\Phi_{\mathrm{p}}(t)$ for the three configurations are summarised in Table II. The values for PR- 05 tend to be slightly higher than the other two configurations, but overall the results are very close.

The most conspicuous differences between the three configurations are visible in Fig. 4: the gradient of the plasma potential $\nabla_{r} \Phi_{\mathrm{p}}^{-}$in the powered sheath and the electric potential at the plasma-facing surface of the discharge chamber wall $\Phi_{\text {wall }}^{-}$during the negative peak. These differences are in response to the different extraneous impedances in the RF circuit, in this case the capacitance $C_{\text {wall }}$ of the dielectric

TABLE II. $\Phi_{\mathrm{p}}$ parameters (V).

\begin{tabular}{lcccc}
\hline \hline Config. & $\Phi_{\mathrm{p}}^{+}$ & $\Phi_{\mathrm{p}}^{-}$ & $\left|\Phi_{\mathrm{p}}^{+}-\Phi_{\mathrm{p}}^{-}\right|$ & $\Phi_{\mathrm{p}}$ \\
\hline PR-05 & 64 & 12.8 & 51 & 28.8 \\
PR-10 & 61 & 12.0 & 49 & 27.0 \\
PR-15 & 60 & 12.3 & 48 & 26.8 \\
\hline \hline
\end{tabular}


discharge chamber wall, described earlier in Sec. II. Ions falling through the powered sheath at the negative peak of the RF cycle are accelerated to very high velocities by the electric field $E=-\nabla_{r} \Phi_{\mathrm{p}}^{-}$; therefore, differences in the gradient of $\Phi_{\mathrm{p}}^{-}$or the magnitude of the potential drop in the powered sheath are expected to shift the position of the high energy peak of the IED in the powered sheath. Since $\Phi_{\mathrm{p}}^{+}$is unaffected by the change in the dielectric wall capacitance, the low energy peak of the IED in the powered sheath is expected to remain constant. Since the dimensions of the PR discharge chamber are too small to admit a RFEA or other invasive probes, the only way to gain valuable insight into the ion dynamics in the powered sheath is through simulation.

\section{Dielectric wall surface potential}

Figure 5 plots the electric potential on the plasma-facing surface of the dielectric discharge chamber wall $\Phi_{\text {wall }}(t)$ at the coordinates $(z, r)=(-9.9,2.1)$ in PR-05 (blue solid line), PR-10 (green solid line), and PR-15 (red solid line). Also plotted on the same scale is the electric potential in the powered electrode $\Phi_{\mathrm{pwr}}(t)$ (magenta solid line). The supplied $\mathrm{RF}$ waveform is the same for all three configurations. Only 4 of the total $10 \mathrm{RF}$ cycles of the final results are shown for clarity. The dashed lines denote the mean of each waveform over all $10 \mathrm{RF}$ cycles. Figure 5 clearly shows the asymmetric response of $\Phi_{\text {wall }}(t)$ to the different discharge chamber wall impedances. These $\Phi_{\text {wall }}(t)$ profiles suggest for the powered sheath an IED with a constant low energy peak given the constant maxima $\Phi_{\text {wall }}^{+}$and a high energy peak that is shifted depending on the value of the minima $\Phi_{\text {wall }}^{-}$.

For clearer comparison of the shapes of the $\Phi_{\text {wall }}(t)$ waveforms, Fig. 6 plots $\Phi_{\text {wall }}^{\prime}(t)=\Phi_{\text {wall }}(t)-\Phi_{\text {wall }}$, or the original waveform relative to its mean, alongside $\Phi_{\mathrm{pwr}}(t)$ (magenta solid line). The profiles have a similar shape across PR-05 (blue solid line), PR-10 (green solid line), and PR-15 (red solid line). $\Phi_{\text {wall }}^{\prime}(t)$ mostly preserves the sinusoidal waveform of $\Phi_{\mathrm{pwr}}(t)$ but is asymmetric about its mean, with a diminished trailing edge at each positive

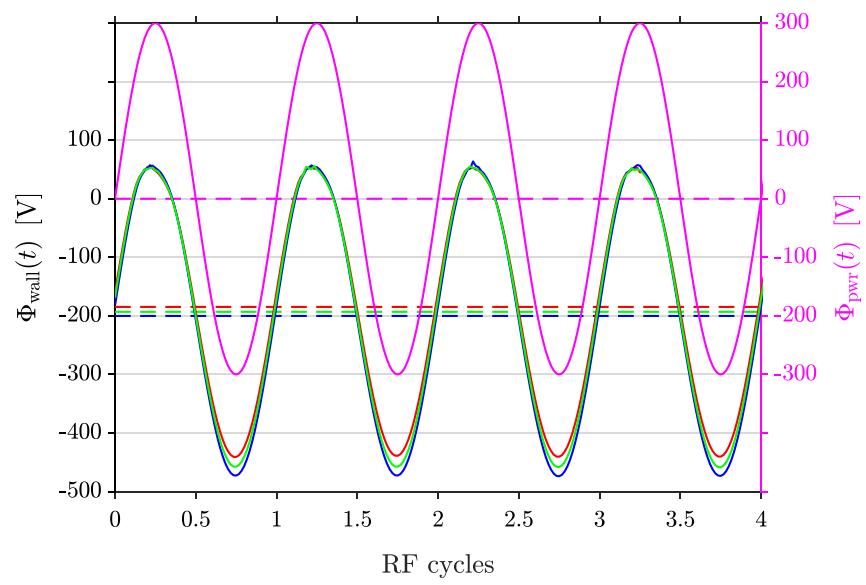

FIG. 5. Electric potential on the plasma-facing surface of the dielectric discharge chamber wall $\Phi_{\text {wall }}(t)$ in PR-05 (blue solid line), PR-10 (green solid line), and PR-15 (red solid line), and the electric potential in the powered electrode $\Phi_{\mathrm{pwr}}(t)$ (magenta solid line). Dashed lines denote the mean values. $\Phi_{\text {wall }}(t)$ responds asymmetrically to the different discharge chamber wall impedances, affecting only the negative peaks and minima $\Phi_{\text {wall }}^{-}$.

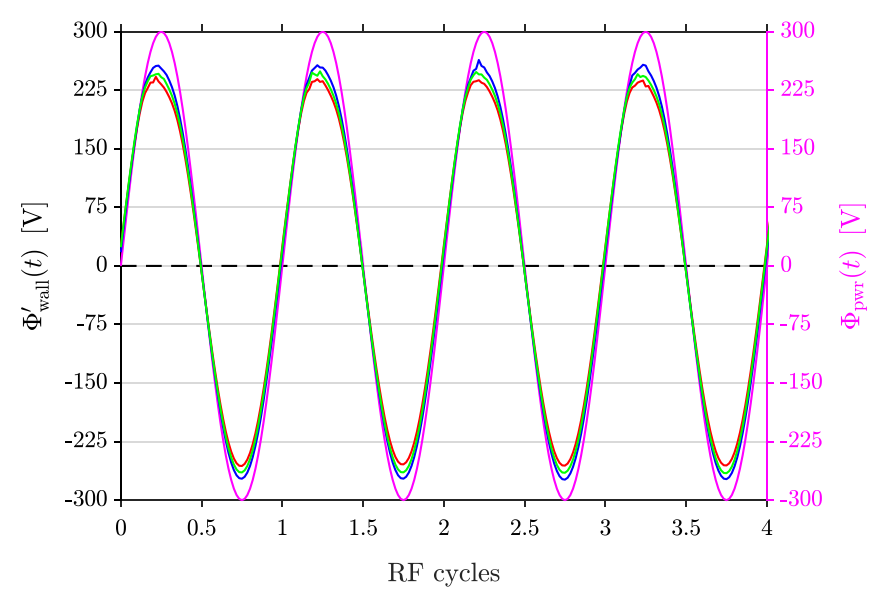

FIG. 6. When plotting $\Phi_{\text {wall }}(t)$ relative to its mean, $\Phi_{\text {wall }}^{\prime}(t)$ across the three configurations mostly preserve the sinusoidal waveform of $\Phi_{\text {pwr }}(t)$ but are asymmetric with a diminished trailing edge at each positive peak.

peak. ${ }^{12}$ The peak-to-peak voltages are the greatest in PR05 and least in PR-15.

Table III lists the maxima, minima, peak-to-peak, and mean values of $\Phi_{\text {wall }}(t)$ for each configuration, including the degree of variation in each parameter. $\Phi_{\text {wall }}^{-}$in the second column of Table III quantifies the asymmetric response exhibited by the negative peaks in the $\Phi_{\text {wall }}(t)$ waveforms to different extraneous impedances in the RF circuit, in this case, the dielectric wall capacitance, described earlier in Sec. II and visible in Figs. 4 and 5. In PR, the affected parameter is $\Phi_{\text {wall }}(t)$ instead of $\Phi_{\text {pwr }}(t)$ because the plasma is in contact with the discharge chamber wall instead of the powered electrode. Essentially, the RF circuit in PR has the position of the blocking capacitor (representing the dielectric wall) and the powered electrode switched compared to the typical arrangement. Since the self-bias manifests in $\Phi_{\text {wall }}(t)$ after the powered electrode, $\Phi_{\mathrm{pwr}}(t)$ maintains the electric potential of the supplied RF waveform.

Of particular interest is the alignment of the positive peaks of $\Phi_{\text {wall }}(t)$ for all three configurations in FIG, 5. The positive peaks have an average maximum of $\Phi_{\text {wall }}^{+}=53 \mathrm{~V}$ with a standard deviation of $\sigma\left(\Phi_{\text {wall }}^{+}\right)=3 \mathrm{~V}$, and $\tau_{+} / \tau_{-}=14 / 46$ (out of 60 time-steps) is approximately constant. Since $A_{\mathrm{gnd}} / A_{\mathrm{pwr}}$ is unchanged, (3) implies that $u_{\mathrm{B}} n_{\mathrm{i}, \mathrm{pwr}} / u_{\mathrm{B}} n_{\mathrm{i} \text {,gnd }}$ is approximately constant in each of the three configurations. While it is possible to verify this by integrating over all the plasma-facing powered (discharge chamber wall) and grounded electrode surfaces over each RF cycle with the present simulation results, it is a nontrivial task given the size of the dataset and the presence of other non-electrode surfaces in PR.

Nonetheless, Figs. 7 and 8 are provided for reference. Figure 7 plots the mean ion density $n_{\mathrm{i}}$ (solid lines) and the mean radial ion drift velocity $u_{r, \mathrm{i}}$ (dashed-dotted lines)

TABLE III. $\Phi_{\text {wall }}$ parameters [V].

\begin{tabular}{ccccc}
\hline \hline Config. & $\Phi_{\text {wall }}^{+}$ & $\Phi_{\text {wall }}^{-}$ & $\left|\Phi_{\text {wall }}^{+}-\Phi_{\text {wall }}^{-}\right|$ & $\Phi_{\text {wall }}$ \\
\hline PR-05 & 56 & -472 & 528 & -200 \\
PR-10 & 53 & -458 & 511 & -193 \\
PR-15 & 52 & -441 & 494 & -185 \\
\hline \hline
\end{tabular}




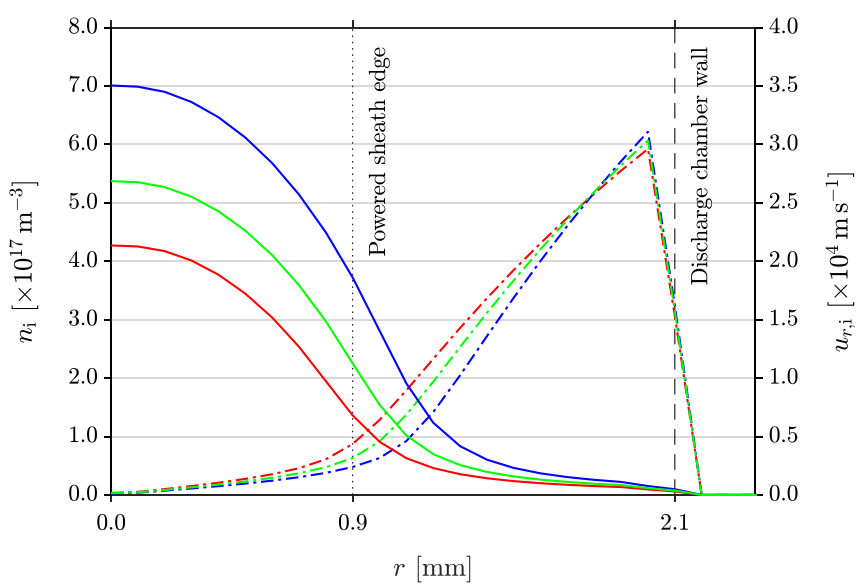

FIG. 7. Radial profile along $z=-9.9$ of the mean ion density $n_{\mathrm{i}}$ (solid lines) and the mean radial ion drift velocity $u_{r, \mathrm{i}}$ (dashed-dotted lines) in the discharge chamber of PR-05 (blue), PR-10 (green), and PR-15 (red). Note that the inner radius of the discharge chamber is kept constant at $2.1 \mathrm{~mm}$ and only the external radius is changed in PR- 05 and PR-15.

radially along $z=-9.9$ under the powered electrode in the discharge chamber, over the $10 \mathrm{RF}$ cycles in the final solution, in PR-05 (blue), PR-10 (green), and PR-15 (red). $n_{\mathrm{i}}$ peaks on the $z$-axis and falls sharply in the powered sheath as $u_{r, \mathrm{i}}$ increases to $u_{r, \mathrm{i}}=3.0 \times 10^{4} \mathrm{~m} \mathrm{~s}^{-1}$ due to the steep potential drop from $\Phi_{\mathrm{p}}$ to $\Phi_{\text {wall }}$ across the powered sheath. ${ }^{31}$ Figure 8 plots the mean axial ion density $n_{\mathrm{i}}$ (solid lines) and the mean axial ion drift velocity $u_{z, \mathrm{i}}$ (dashed-dotted lines) along the $z$-axis at $r=0$ using the same colours for each configuration. The position of the powered electrode is shown by the brown bar at the top. $n_{\mathrm{i}}$ peaks under the powered electrode at the coordinates $(z, r)=(-9.9,0)$ in all three configurations, with similarly shaped profiles featuring a strong central gamma mode peak and two shoulder alpha mode plateaus. The peak values are $n_{\mathrm{i}}=7.0 \times 10^{17} \mathrm{~m}^{-3}$, $5.4 \times 10^{17} \mathrm{~m}^{-3}$, and $4.3 \times 10^{17} \mathrm{~m}^{-3}$ for PR-05, PR-10, and $\mathrm{PR}-15$, respectively. The left edge of the plot at $z=-30$ is the grounded electrode, where ions impact onto the front wall of the plenum at $u_{z, \mathrm{i}}=-5.0 \times 10^{3} \mathrm{~m} \mathrm{~s}^{-1}$. Along the

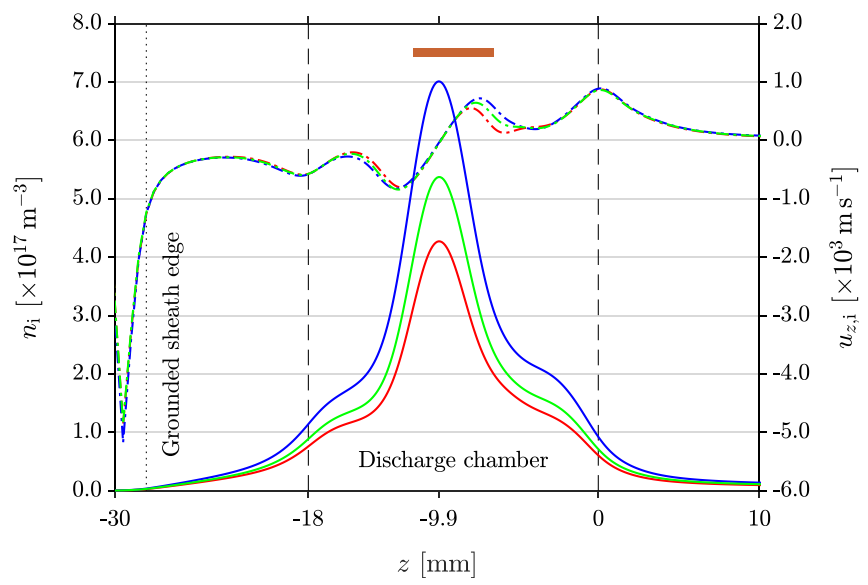

FIG. 8. Axial profile of the mean ion density $n_{\mathrm{i}}$ (solid lines) and the mean axial ion drift velocity $u_{z, \mathrm{i}}$ (dashed-dotted lines) along the $z$-axis for PR- 05

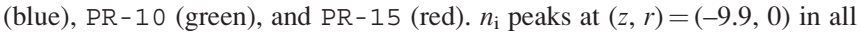
three configurations, with similarly shaped profiles featuring a strong central gamma mode peak and two shoulder alpha mode plateaus. $z$-axis, ions are on average moving away from the central $n_{\mathrm{i}}$ peak, which is expected as the plasma potential $\Phi_{\mathrm{p}}$ is most positive at the peak. Figures 7 and 8 show a consistent $u_{r, i} n_{\mathrm{i}, \mathrm{pwr}} / u_{z, \mathrm{i}} n_{\mathrm{i}, \text { gnd }}$ across the three configurations in the powered sheath and front wall grounded sheath, respectively, which is a reasonable indication of similar behaviour for the plasma sheaths at all of the grounded electrode surfaces in general. Regardless, the high degree of alignment of the positive peaks and the preservation of a constant $\tau_{+} / \tau_{-}$in Fig. 5 are sufficient evidence to prove it so.

The final assumptions to be verified before calculating the self-bias voltage $V_{\text {bias }}$ are the ion transit time $\tau_{\mathrm{i}}$ and the variation in the ion drift velocity $\Delta u_{r, \mathrm{i}}$ in the collisional powered sheath. The former is calculated by performing a fourth order Runge-Kutta analysis on $u_{r, \mathrm{i}}$ to obtain $r_{\mathrm{i}}$, the mean radial drift position of the ion as a function of time. ${ }^{31}$ The transit times are $\tau_{\mathrm{i}}=134 \mathrm{~ns}=1.8 \tau_{\mathrm{RF}}$ in PR $-05, \tau_{\mathrm{i}}=103 \mathrm{~ns}$ $=1.4 \tau_{\mathrm{RF}}$ in $\mathrm{PR}-10$, and $\tau_{\mathrm{i}}=86 \mathrm{~ns}=1.2 \tau_{\mathrm{RF}}$ in PR-15. Given that $\tau_{\mathrm{i}} \sim \tau_{\mathrm{RF}}, \Delta u_{r, \mathrm{i}}$ is expected to be somewhat similar across the three configurations: $\Delta u_{r, \mathrm{i}}= \pm 29.9 \%, \pm 27.1 \%$, and $\pm 24.6 \%$ in PR-05, PR-10, and PR-15, respectively. Since $\Delta u_{r, \mathrm{i}}$ is significant for all three configurations, the assumptions made for the $\tau_{\mathrm{i}} \ll \tau_{\mathrm{RF}}$ regime are reasonably valid for PR.

\section{Self-bias voltage}

Finally, substituting $\tau+/ \tau-=14 / 46$ into (4) gives

$$
V_{\text {bias }}=-V_{\mathrm{RF}} \sin \left(\frac{\pi}{2} \cdot \frac{46-14}{46+14}\right)=-0.743 V_{\mathrm{RF}}
$$

which for $V_{\mathrm{RF}}=300 \mathrm{~V}$ results in $V_{\text {bias }}=-223 \mathrm{~V}$. However, (4) is only accurate for a plasma that is in direct electrically conductive contact with the powered electrode and exposed to the full $V_{\mathrm{RF}}$ amplitude of $\Phi_{\mathrm{pwr}}(t)$. This is no longer true in plasma systems where the powered electrode is shielded from the plasma by a component with a non-negligible impedance. A portion of the RMS RF voltage is dropped across the component, and the resultant RF voltage amplitude on the plasma is $<V_{\mathrm{RF}}$. In $\mathrm{PR}$, this component is the capacitive discharge chamber wall. In an industrial RIE system, this is the wafer impedance, which includes wafer bulk and spreading resistances, oxide and insulating film capacitances, as well as gap capacitance and contact resistance between the wafer and the powered electrode chuck. ${ }^{47}$ The plasma in these systems is exposed to $\Phi_{\text {wall }}(t)$ or $\Phi_{\text {wafer }}(t)$, which have amplitudes $<V_{\mathrm{RF}}$.

For this type of shielded plasma system, in particular those operating in the $\tau_{\mathrm{i}} \leqslant \tau_{\mathrm{RF}}$ regime, $V_{\text {bias }}$ can be defined in a manner that is unaffected by the extra impedances between the powered electrode and the plasma, by using the maxima envelope of the waveform on the plasma-facing surface of the shielding component

$$
V_{\text {bias }}^{\prime}=\Phi_{\text {shield }}^{+}-V_{\mathrm{RF}} .
$$

This definition of $V_{\text {bias }}^{\prime}$ is straightforward and applicable to non-sinusoidal waveforms in general. Most importantly, it is rigorous since $\Phi_{\text {shield }}^{+}$is directly correlated with the 
measurable low energy peak of the IED in the powered sheath. $V_{\text {bias }}^{\prime}$ from (8) may also be compared with $V_{\text {bias }}$ from (4) to quantify any deviances of the plasma system from the ideal case. For PR, (8) takes advantage of the asymmetric response of $\Phi_{\text {wall }}(t)$ to the different discharge chamber wall impedances. The result is a constant $V_{\text {bias }}^{\prime}=\Phi_{\text {wall }}^{+}-V_{\mathrm{RF}}$ $=53 \mathrm{~V}-300 \mathrm{~V}=-247 \mathrm{~V}$ across PR-05, PR-10, and PR-15, which is expected as the root cause of the self-bias, the geometrical area asymmetry of the grounded and powered electrodes, is the same across all three configurations.

Note that using the traditional definition of $V_{\text {bias }}^{\prime \prime}=\Phi_{\text {wall }}$ is misleading as it gives erroneous and different values (last column in Table III) for each configuration despite them having a constant $A_{\text {gnd }} / A_{\text {pwr }}$ area ratio. Moreover, $V_{\text {bias }}^{\prime \prime}$ is not meaningful as there is no direct correlation between its value and the discharge chamber wall impedances and does not give meaningful insight into the IED in the powered sheath. In earlier works ${ }^{41,44}$ where the imposed voltage on the powered electrode is $V_{\mathrm{RF}}=240 \mathrm{~V}, \Phi_{\text {wall }}(t)$ is reported to oscillate between $\Phi_{\text {wall }}^{+}=70 \mathrm{~V}$ and $\Phi_{\text {wall }}^{-}=-180 \mathrm{~V}$. This means that the RMS RF voltage drop across the discharge chamber wall is $81 \mathrm{~V}$, which is a significant $48 \%$ of the original $170 \mathrm{~V}$. The raised negative peaks of $\Phi_{\text {wall }}(t)$ due to such a drastic attenuation cause the mean $\Phi_{\text {wall }}$ to be artificially raised. This ultimately results in an erroneous $V_{\text {bias }}^{\prime \prime}=-33 \mathrm{~V}$, which is unphysical as it is much smaller than the difference in the electric potentials $\Phi_{\text {wall }}^{+}-V_{\mathrm{RF}}=-170 \mathrm{~V}$ during the positive peaks of the RF cycle. These issues have been remedied in the present generation of simulations and results.

If $V_{\text {bias }}^{\prime}$ is constant, then the self-bias charge $Q_{\text {bias }}$ $=C_{\text {wall }} V_{\text {bias }}^{\prime}$ that accumulates on the capacitive discharge chamber wall must be proportional to $C_{\text {wall }}$. The negative charging of the discharge chamber wall happens during plasma breakdown and occurs on a time scale of several RF cycles, limited by the speed of positive ions flowing into the grounded electrode. ${ }^{18}$ While the CFD-plasma simulations do indeed capture the self-bias charging behaviour during this period, the solutions in the beginning are still evolving from the initial conditions and are far from being converged. Hence, the data required to calculate $Q_{\text {bias }}$ are not explicitly available. However, $Q_{\text {bias }} \propto C_{\text {wall }} \propto n_{\mathrm{i}}$ is a reasonable estimate if the charging duration during plasma breakdown is roughly similar across the three configurations. The values of $C_{\text {wall }}$ and $n_{\mathrm{i}}$ given in Secs. V B and VC respectively, are positively correlated and therefore support this estimate.

\section{E. Power and voltage division}

As shown in Figs. 7 and 8, the mean ion density $n_{\mathrm{i}}$ is the highest in PR-05 and the lowest in PR-15. It is known from the plasma energy balance equation ${ }^{20}$ that $n_{\mathrm{i}}$ is dependent on the total power absorbed by the plasma. The steady state power $\bar{P}$ may be calculated by integrating the volumetric and surface energy in the PR simulation domain over the $10 \mathrm{RF}$ cycles in the final solution. This gives $\bar{P}=7.0 \mathrm{~W}$, $5.0 \mathrm{~W}$, and $3.8 \mathrm{~W}$ in PR-05, PR-10, and PR-15, respectively. $\bar{P}$ for $\mathrm{PR}-10$ is in close agreement with the value of $4.8 \mathrm{~W}$ measured with a digital inline voltage/current (V/I) probe on the PR experimental setup. The mean ion density peaks have the respective values: $n_{\mathrm{i}}=7.0 \times 10^{17} \mathrm{~m}^{-3}, 5.4$ $\times 10^{17} \mathrm{~m}^{-3}$, and $4.3 \times 10^{17} \mathrm{~m}^{-3}$, which has a linear correlation coefficient of 0.9995 with $\bar{P}$ using these three data points. The shape of the $n_{\mathrm{i}}$ profiles is also in good agreement with Langmuir probe experimental measurements. ${ }^{7}$

Figure 9 plots $\Delta \Phi_{\text {wall }}(t)=\left|\Phi_{\text {pwr }}(t)-\Phi_{\text {wall }}^{\prime}(t)\right|$, the absolute RF potential difference across the dielectric wall after correcting for the mean negative DC bias of $\Phi_{\text {wall }}$. The profiles are similarly shaped across PR-05 (blue solid line), PR-10 (green solid line), and PR-15 (red solid line). The asymmetric waveform is highlighted by the leading peaks resulting from a greater RF potential difference between $\Phi_{\mathrm{pwr}}(t)$ and the diminished trailing edge of the positive peaks in $\Phi_{\text {wall }}^{\prime}(t)$. The asymmetry also causes the points of intersection between the two waveforms to be displaced from the expected times at every $0.5 \mathrm{RF}$ cycle. The RMS values of the $\Delta \Phi_{\text {wall }}(t)$ profiles (dashed line) are $\bar{V}_{\text {wall }}=26 \mathrm{~V}, 33 \mathrm{~V}$, and $39 \mathrm{~V}$ for PR-05, PR-10, and PR-15, respectively.

Similar calculations can also be performed for the powered and grounded sheaths by tracking the RF potential difference across the widths of the sheaths. For the powered sheath, $\quad \Delta \Phi_{\mathrm{s}, \mathrm{pwr}}(t)=\left|\Phi_{\mathrm{p}, \mathrm{pwr}}^{\prime}(t)-\Phi_{\mathrm{wall}}^{\prime}(t)\right| \quad$ is evaluated between the inner edge of the powered sheath at the coordinates $(z, r)=(-9.9,0.9)$ and the plasma-facing surface of the discharge chamber wall at $(-9.9,2.1)$. For the grounded sheath, $\Delta \Phi_{\mathrm{s}, \text { gnd }}(t)=\left|\Phi_{\mathrm{p}, \text { gnd }}^{\prime}(t)-\Phi_{\mathrm{gnd}}^{\prime}(t)\right|$ is evaluated between the inner edge of the grounded sheath at the coordinates $(z$, $r)=(-28.06,0)$ and the plasma-facing surface of the front wall at $(-30,0)$. The RMS values of $\Delta \Phi_{\mathrm{s}, \mathrm{pwr}}(t)$ are $\bar{V}_{\mathrm{s} \text {,pwr }}$ $=176 \mathrm{~V}, 171 \mathrm{~V}$, and $164 \mathrm{~V}$ for PR-05, PR-10, and PR-15, respectively, while the RMS values of $\Delta \Phi_{\mathrm{s} \text {,gnd }}(t)$ are $\bar{V}_{\mathrm{s}, \text { gnd }}=9.2 \mathrm{~V}, 8.0 \mathrm{~V}$, and $7.7 \mathrm{~V}$ given in the same order. Note that $\bar{V}_{\text {wall }}+\bar{V}_{\mathrm{s}, \mathrm{pwr}}+\bar{V}_{\mathrm{s} \text {,gnd }}$ do not exactly add up to $\bar{V}_{\mathrm{RF}}=212.1 \mathrm{~V}$ due to the irregular shapes of the waveforms, but the errors are only on the order of $\sim 1 \mathrm{~V}$.

The $\bar{V}_{\mathrm{s}, \mathrm{pwr}}$ and $\bar{V}_{\mathrm{s}, \text { gnd }}$ values may be checked against the powered and grounded sheath impedances calculated earlier in Sec. V B. The capacitive discharge chamber wall, powered

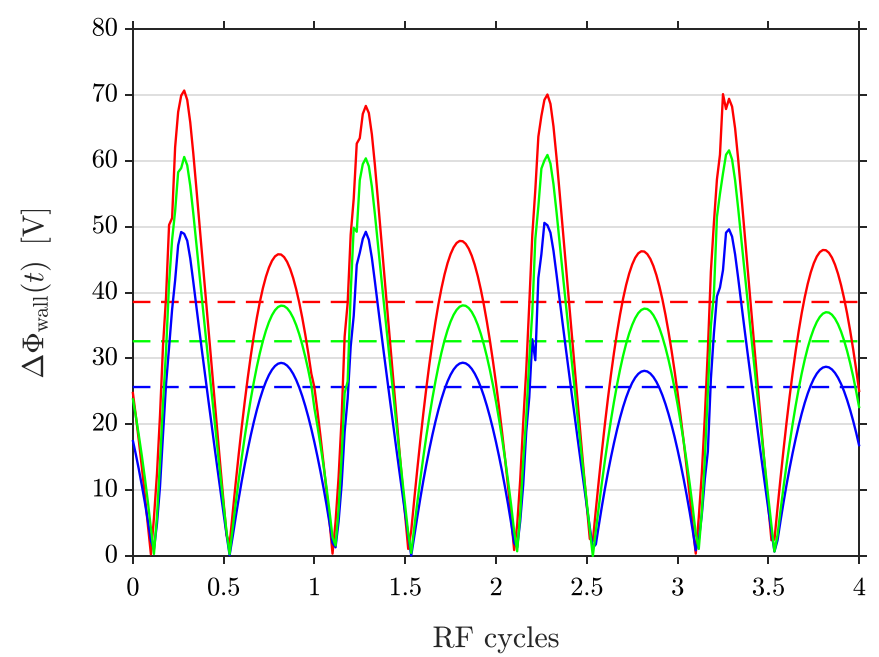

FIG. 9. Absolute RF potential difference across the dielectric wall $\Delta \Phi_{\text {wall }}(t)$ in PR- 05 (blue solid line), PR-10 (green solid line), and PR-15 (red solid line). The dashed-dotted lines denote the RMS voltage $\bar{V}_{\text {wall }}$ of each profile. Only four RF cycles are shown for clarity. 
sheath, and grounded sheath may be represented by a voltage divider circuit with their respective impedances $\left|Z_{\text {wall }}\right|$, $\left|Z_{\text {s,pwr }}^{\prime}\right|$, and $\left|Z_{\text {s,gnd }}^{\prime}\right|$ in series between $\bar{V}_{\mathrm{RF}}=212.1 \mathrm{~V}$ and ground. However, due to the unknown proportionality constant $z$, the powered and grounded sheath section of the voltage divider circuit must be considered separately from the capacitive discharge chamber wall.

For convenience of calculation, assume a sinusoidal waveform for $\Delta \Phi_{\text {wall }}(t)$ instead of its actual shape shown in Fig. 9. Then, $\bar{V}_{\mathrm{RF}}-\bar{V}_{\text {wall }}^{\prime}=\bar{V}_{\mathrm{p}}^{\prime}$ gives the total RMS RF voltage across both sheaths. Thus, the RMS RF voltage drop across each sheath in a voltage divider circuit is given by

$$
\bar{V}_{\mathrm{s}}^{\prime}=\bar{V}_{\mathrm{p}}^{\prime} \frac{\left|Z_{\mathrm{s}}^{\prime}\right|}{\left|Z_{\mathrm{p}}^{\prime}\right|},
$$

where $\left|Z_{\mathrm{s}}^{\prime}\right|$ represents either $\left|Z_{\mathrm{s}, \mathrm{pwr}}^{\prime}\right|$ or $\left|Z_{\mathrm{s}, \text { gnd }}^{\prime}\right|,\left|Z_{\mathrm{p}}^{\prime}\right|=\left|Z_{\mathrm{s}, \mathrm{pwr}}^{\prime}\right|$ $+\left|Z_{\mathrm{s}, \text { gnd }}^{\prime}\right|$, and the proportionality constant $z$ is eliminated. With $\left|Z_{\mathrm{s}, \mathrm{pwr}}^{\prime}\right| \approx z \cdot 3.7 \mathrm{k} \Omega$ and $\left|Z_{\mathrm{s}, \mathrm{gnd}}^{\prime}\right| \approx z \cdot 51 \Omega$, the RMS RF voltage drop across the powered sheath is $\bar{V}_{\mathrm{s}, \mathrm{pwr}}^{\prime}=184 \mathrm{~V}$, $177 \mathrm{~V}$, and $171 \mathrm{~V}$ for $\mathrm{PR}-05, \mathrm{PR}-10$, and $\mathrm{PR}-15$, respectively, while the RMS RF voltage drop across the grounded sheath is $\bar{V}_{\mathrm{s} \text {,gnd }}^{\prime}=2.5 \mathrm{~V}, 2.4 \mathrm{~V}$, and $2.4 \mathrm{~V}$ in the same order. In this case, the RMS RF voltages sum up to $\bar{V}_{\text {wall }}+\bar{V}_{\text {s,pwr }}^{\prime}$ $+\bar{V}_{\mathrm{s}, \text { gnd }}^{\prime}=\bar{V}_{\mathrm{RF}}=212.1 \mathrm{~V}$. These values are summarised in Table IV.

The most obvious discrepancy is $\bar{V}_{\mathrm{s} \text {,gnd }}^{\prime}$ being much lower than $\bar{V}_{\mathrm{s} \text {,gnd }}$. This is due to the calculation of $\left|Z_{\mathrm{s}, \mathrm{gnd}}^{\prime}\right|$ and $C_{\mathrm{s} \text {,gnd }}^{\prime}$ not taking into account the variation in distance between the grounded sheath and the plasma bulk due to the geometry of the plenum and the end wall. Areas of the grounded sheath further away from the plasma bulk and the powered electrode are less affected by the oscillating RF potential. The reduction in the effective area results in a smaller $C_{\mathrm{s} \text {,gnd }}^{\prime}$ and therefore a larger $\left|Z_{\mathrm{s}, \mathrm{gnd}}^{\prime}\right|$, which then increases the share of $\bar{V}_{\mathrm{s}, \mathrm{gnd}}^{\prime}$ and decreases $\bar{V}_{\mathrm{s} \text {,pwr }}^{\prime}$. Note that $\bar{V}_{\text {s,pwr }}$ and $\bar{V}_{\text {s,gnd }}$ are not significantly affected by geometry as they are measured along closely adjacent electric field lines. Overall, the two methods of calculating the RMS RF voltage division are in good agreement.

With these values of $\bar{V}_{\text {wall }}, \bar{V}_{\text {s,pwr }}$, and $\bar{V}_{\text {s,gnd }}$, it is now possible to calculate the equivalent powered and grounded sheath impedances using

$$
\left|Z_{\mathrm{s}}\right|=\left|Z_{\text {wall }}\right| \frac{\bar{V}_{\mathrm{s}}}{\bar{V}_{\text {wall }}} .
$$

This gives $\left|Z_{\mathrm{s}, \mathrm{pwr}}\right|=6.4 \mathrm{k} \Omega, 9.0 \mathrm{k} \Omega$, and $10.1 \mathrm{k} \Omega$ and $\left|Z_{\mathrm{s}, \text { gnd }}\right|=0.34 \mathrm{k} \Omega, 0.42 \mathrm{k} \Omega$, and $0.47 \mathrm{k} \Omega$ for PR-0 5, PR-10, and $\mathrm{PR}-15$, respectively. Following on from this, it is now possible to calculate the RMS RF current via Ohm's law,

TABLE IV. $\bar{V}$ and $\bar{V}^{\prime}$ values [V].

\begin{tabular}{lccccc}
\hline \hline Config. & $\bar{V}_{\text {wall }}$ & $\bar{V}_{\text {s,pwr }}$ & $\bar{V}_{\text {s,pwr }}^{\prime}$ & $\bar{V}_{\text {s,gnd }}$ & $\bar{V}_{\text {s,gnd }}^{\prime}$ \\
\hline PR-05 & 26 & 176 & 184 & 9.2 & 2.5 \\
PR-10 & 33 & 171 & 177 & 8.0 & 2.4 \\
PR-15 & 39 & 164 & 171 & 7.7 & 2.4 \\
\hline \hline
\end{tabular}

which gives $\bar{I}_{\mathrm{RF}}=27 \mathrm{~mA}, 19 \mathrm{~mA}$, and $16 \mathrm{~mA}$ for the three configurations. The accuracy of these values may be checked by calculating the power $\bar{P}_{\mathrm{RF}}=\bar{V}_{\mathrm{RF}} \bar{I}_{\mathrm{RF}}$, which gives $5.8 \mathrm{~W}$, $4.0 \mathrm{~W}$, and $3.5 \mathrm{~W}$ for PR-05, PR-10, and PR-15, respectively. $\bar{P}_{\mathrm{RF}}$ excludes the power sunk into non-electrical processes such as neutral gas heating ${ }^{31}$ and chemical reactions and thus is in good agreement with the integrated steady state power $\bar{P}$ for each configuration listed earlier in this section.

\section{F. Powered electrode current}

By Ohm's law, the RMS voltage drop across the capacitive discharge chamber wall is equal to the product of the RMS current and its impedance or $\bar{V}_{\text {wall }}=\bar{I}_{\text {wall }}\left|Z_{\text {wall }}\right|$. However, obtaining and integrating the spatiotemporal electrical current in PR are nontrivial, so the following is an attempt to estimate $\bar{I}_{\text {wall }}$, the RMS current at the plasmafacing surface of the discharge chamber wall directly underneath the powered electrode.

$I_{\text {wall }}(t)$ is a combination of three different charged species fluxes: (i) $I_{\mathrm{i}}(t)$ from the ion flux, which is continuous DC into the wall with a small varying $\Delta u_{r, \mathrm{i}}$ over it, (ii) $I_{\mathrm{e}}(t)$ from the primary electron flux, which is a large diodic spike into the wall during $\tau_{+}$, and (iii) $I_{\mathrm{e}^{\prime}}(t)$ from the secondary electron flux, which is a diodic current proportional to the ion flux by the SEEC, out of the wall during $\tau_{-}$. These currents are visualised in Fig. 10. $\Phi_{\text {wall }}(t)$ (black dashed-dotted line) is plotted showing the positive period $\tau_{+}$and the negative period $\tau_{-}$on the horizontal axis in the RF cycle. The vertical axes are normalised to the mean $I_{\mathrm{i}}=1$ (blue dashed line), with $I_{\mathrm{i}}(t)$ (blue) assumed to have a sinusoidal variation of amplitude $\Delta u_{r, \mathrm{i}}$ on top of $I_{\mathrm{i}}$, in phase with $-\Phi_{\text {wall }}(t)$. The simulations use SEEC $=0.1$, which means that $I_{\mathrm{e}^{\prime}}(t)$ $=0.1 \cdot I_{\mathrm{i}}(t)$ (magenta), but only during $\tau_{-} . I_{\mathrm{i}}(t)$ and $I_{\mathrm{e}^{\prime}}(t)$ are positive as they represent a positive ion current into the wall and a negative secondary electron current out of the wall, respectively. Over one RF cycle, the total negative charge collected by the wall at the powered electrode must be equal to the total positive charge (1). Hence, the negative area bound by $I_{\mathrm{e}}(t)$ (red) must be equal to the positive area bound by $I_{\mathrm{i}}(t)+I_{\mathrm{e}^{\prime}}(t)$ over $\tau_{\mathrm{RF}}$. Since $I_{\mathrm{e}}(t)$ is limited to $\tau_{+}$, its vertical amplitude is much larger at $I_{\mathrm{e}}^{-}=-7.3$ approximately the same for all three configurations, assuming a shape resembling a single negative rectified sinusoidal peak. This number is approximately constant for all three configurations as their $\Delta u_{r, \mathrm{i}}$ are quite similar. Thus, $I_{\text {wall }}(t)=I_{\mathrm{i}}(t)+I_{\mathrm{e}}(t)+I_{\mathrm{e}^{\prime}}(t)$ is the black solid line in Fig. 10, giving the total RMS current $\bar{I}_{\text {wall }}=k \cdot \bar{I}_{\mathrm{i}}$, with a proportionality constant $k \approx 2.4$ for all three configurations.

Now with this estimate for $\bar{I}_{\text {wall }}$, Ohm's law in the discharge chamber wall may be roughly approximated as

$$
\bar{V}_{\text {wall }}=a \cdot k \cdot \bar{I}_{\mathrm{i}}\left|Z_{\mathrm{wall}}\right|=a \cdot k \cdot \frac{e \overline{u_{r, \mathrm{i}} n_{\mathrm{i}}} A_{\mathrm{wall}}}{\omega C_{\mathrm{wall}}}
$$

by assuming $\bar{I}_{\mathrm{i}}=e \overline{u_{r, \mathrm{i}} n_{\mathrm{i}}} A_{\text {wall }}$, where $\overline{u_{r, \mathrm{i}} n_{\mathrm{i}}}$ is the RMS ion density flux evaluated at the coordinates $(z, r)=(-9.9,0.9)$ at the inner edge of the powered sheath, and $A_{\text {wall }}$ is the area of the plasma-facing surface of the discharge chamber wall directly underneath the powered electrode. Using $\bar{V}_{\text {wall }}$ from 
Fig. 9 and the values of the various parameters quoted earlier, the dimensionless proportionality constant for (11) is calculated to be $a \approx 1.2,1.1$, and 1.1 for the three respective configurations. The close proximity of $a$ to unity determines that the derivation of $\bar{I}_{\text {wall }}=k \cdot \bar{I}_{\mathrm{i}}$ and (11) are sound and accurate.

\section{G. Secondary electron emission}

Figure 11 plots the temporal variation of the electron energy $k_{\mathrm{B}} T_{\mathrm{e}}(t)$ at the coordinates $(z, r)=(-9.9,2.0)$, in the discharge chamber one cell away from the plasma-facing surface of the discharge chamber wall. This is necessary as the fluid-solid interface is rendered across two cells on either side of the boundary ${ }^{31}$ for certain parameters such as $k_{\mathrm{B}} T_{\mathrm{e}}$ and $u_{r, \mathrm{i}}$ (Fig. 7). The $k_{\mathrm{B}} T_{\mathrm{e}}(t)$ profiles for $\mathrm{PR}-05$ (blue solid line), PR-10 (green solid line), and PR-15 (red solid line) are similar in shape and only differ in height. Also plotted in Fig. 11 is the electric potential $\Phi_{\text {wall }}(t)$ (black dashed-dotted line) at the plasma-facing surface of the discharge chamber wall at the coordinates $(z, r)=(-9.9,2.1)$. Only the positive peaks of $\Phi_{\text {wall }}(t)$ are shown for reference.

During $\tau_{+}$when $\Phi_{\text {wall }}(t)$ is positive, $k_{\mathrm{B}} T_{\mathrm{e}}(t)$ is low at approximately $2.0 \mathrm{eV}$ for all three configurations, indicating the presence of mainly primary electrons from the plasma bulk. During $\tau_{-}$however when $\Phi_{\text {wall }}(t)$ is at its most negative, $k_{\mathrm{B}} T_{\mathrm{e}}(t)$ peaks at $123 \mathrm{eV}, 106 \mathrm{eV}$, and $92 \mathrm{eV}$ for $\mathrm{PR}-05$, $P R-10$, and $P R-15$, respectively, indicating the presence of high energy secondary electrons that have been emitted from the surface of the discharge chamber wall due to ion bombardment. The height of the $k_{\mathrm{B}} T_{\mathrm{e}}(t)$ peak is not only dependent on the magnitude of the potential drop across the powered sheath but also a number of other factors (e.g., RF driving frequency, gradient of the plasma potential, and neutral density) that affect the temporally varying ion drift velocity $u_{r, \mathrm{i}}(t)$ in the collisional powered sheath and the ion bombardment energy. Hence, a fully self-consistent plasma model is required to accurately resolve ion bombardment behaviour and secondary electron emission characteristics in PR.

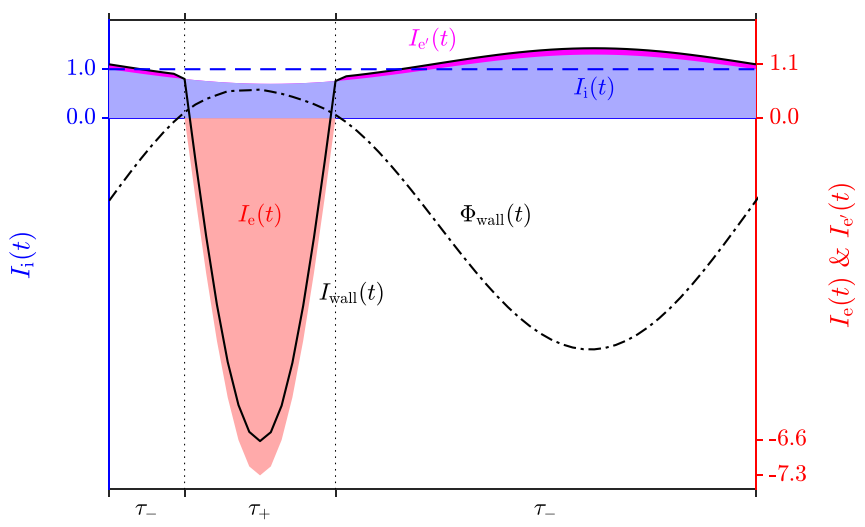

FIG. 10. Representation of the electron current $I_{\mathrm{e}}(t)$ (red), ion current $I_{\mathrm{i}}(t)$ (blue), and secondary electron current $I_{\mathrm{e}^{\prime}}(t)$ (magenta) at the surface of the discharge chamber wall. The total current $I_{\text {wall }}(t)$ (black solid line) is the sum of these individual currents. The vertical axes are normalised to $I_{\mathrm{i}}=1$ (blue dashed line). $\Phi_{\text {wall }}(t)$ (black dashed-dotted line) is also plotted to show the positive period $\tau_{+}$and the negative period $\tau_{-}$in the RF cycle.
These high energy secondary electrons are the primary source of ionisation in the discharge chamber, driving the strong central gamma mode peak in the plasma density (Fig. $8)$. Since the high energy secondary electrons are only emitted during $\tau_{-}$and localised to the region under the powered electrode, the ionisation rate (reaction No. 20 in Table I) is the highest in the middle of the discharge chamber during this time. During $\tau_{+}$, there are no high energy secondary electrons. Instead, bulk electrons from the plenum and the second half of the discharge chamber are drawn towards the positive powered electrode, and the ionisation rate is the highest in the first and last thirds of the discharge chamber where the electric field lines are the tightest, creating the shoulder alpha mode plateaus seen in Fig. 8.

\section{H. Spatiotemporal variation}

The secondary electrons emitted from the discharge chamber wall lose most of their energy as they pass through the powered sheath and end up in the plasma bulk at a stable mean energy of $k_{\mathrm{B}} T_{\mathrm{e}}=2.9 \mathrm{eV}$ on average across the three configurations. Figure 12 plots the spatiotemporal variation of $k_{\mathrm{B}} T_{\mathrm{e}}(t)$ radially along $z=-9.9$ in the discharge chamber for PR-05 (blue lines), PR-10 (green lines), and PR-15 (red lines). The solid line in the middle of each profile denotes the mean $k_{\mathrm{B}} T_{\mathrm{e}}$. Notice that the actual peak of the $k_{\mathrm{B}} T_{\mathrm{e}}$ radial profiles occurs at two or three cells away from the plasma-facing surface of the discharge chamber wall (dashed lines). During $\tau+$, the $k_{\mathrm{B}} T_{\mathrm{e}}(t)$ radial profiles are low and flat across the whole radius of the discharge chamber, indicating the collapse of the powered sheath. During $\tau_{-}$, the $k_{\mathrm{B}} T_{\mathrm{e}}(t)$ radial profiles start to rise near the discharge chamber wall as ion bombardment begins to liberate high energy secondary electrons. As the $k_{\mathrm{B}} T_{\mathrm{e}}(t)$ radial profiles rise, they also start to extend further from the discharge chamber wall, indicating the formation of the powered sheath. The set of 60 lines representing the 60 time-steps per RF cycle illustrates the oscillatory behaviour of $k_{\mathrm{B}} T_{\mathrm{e}}(t)$ and the powered sheath.

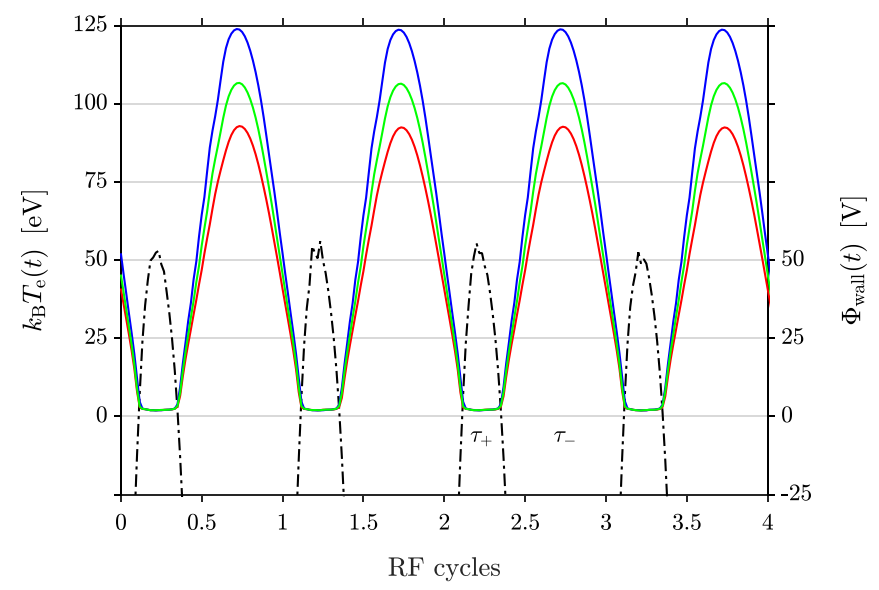

FIG. 11. Temporal variation of the electron energy $k_{\mathrm{B}} T_{\mathrm{e}}(t)$ at $(z, r)=(-9.9$, 2.0 ), in PR- 05 (blue solid line), PR-10 (green solid line), and PR-15 (red solid line). Secondary electrons are emitted only during the negative period $\tau_{-}$of $\Phi_{\text {wall }}(t)$ (black dashed-dotted line). Only four RF cycles are shown for clarity. 


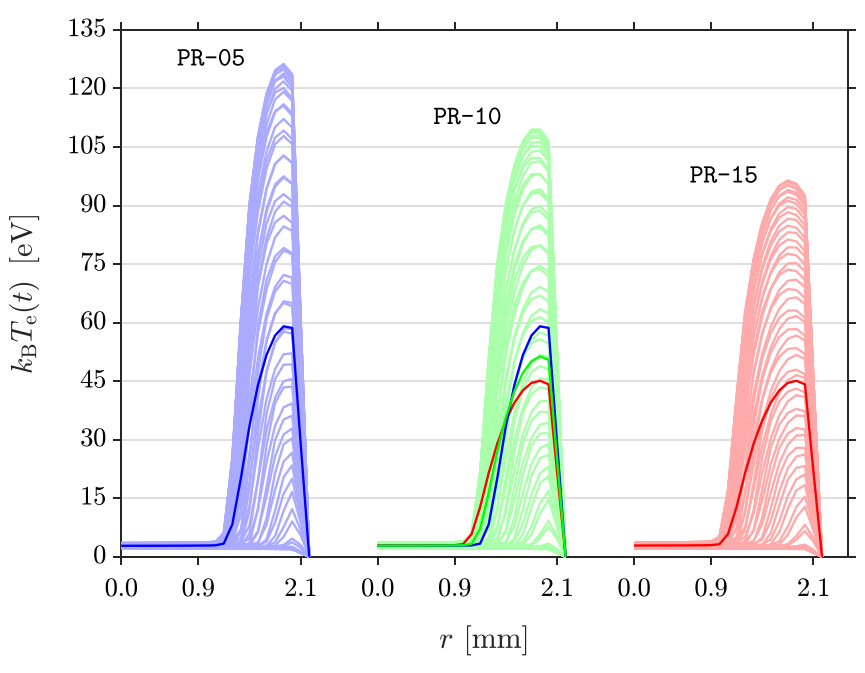

FIG. 12. Spatially and temporally resolved electron energy $k_{\mathrm{B}} T_{\mathrm{e}}(t)$ profiles across the radius of the discharge chamber along $z=-9.9$ in PR- 05 (blue lines), PR-10 (green lines), and PR-15 (red lines). The mean electron energy $k_{\mathrm{B}} T_{\mathrm{e}}$ for PR-05 (blue solid line) and PR-15 (red solid lines) is superimposed onto the $\mathrm{PR}-10$ profile for comparison.

Additionally, there is spatial variation in the distribution of the negative self-bias charge in the discharge chamber wall, mainly localised to the ungrounded section of the wall near the powered electrode. Figure 13 plots the spatiotemporal variation of $\Phi_{\text {wall }}(t)$ (top) axially along $r=2.1$ between $-18 \leq z \leq 0$, and $k_{\mathrm{B}} T_{\mathrm{e}}(t)$ (bottom) axially along $r=2.0$, one cell away from the plasma-facing surface of the discharge chamber wall in the same range. PR-05, PR-10, and PR15 are represented by the colours blue, green, and red, respectively. The solid line in the middle of each plot denotes the mean, while the dotted line shows the temporal variation of each profile over the RF cycle. The axial position of the powered electrode is shown by the brown bar at the top for each configuration, while the rear and end walls of the grounded structure are represented by the grey bars. The uncoloured space between the bars denotes the sections of the discharge chamber wall that are electrically insulated

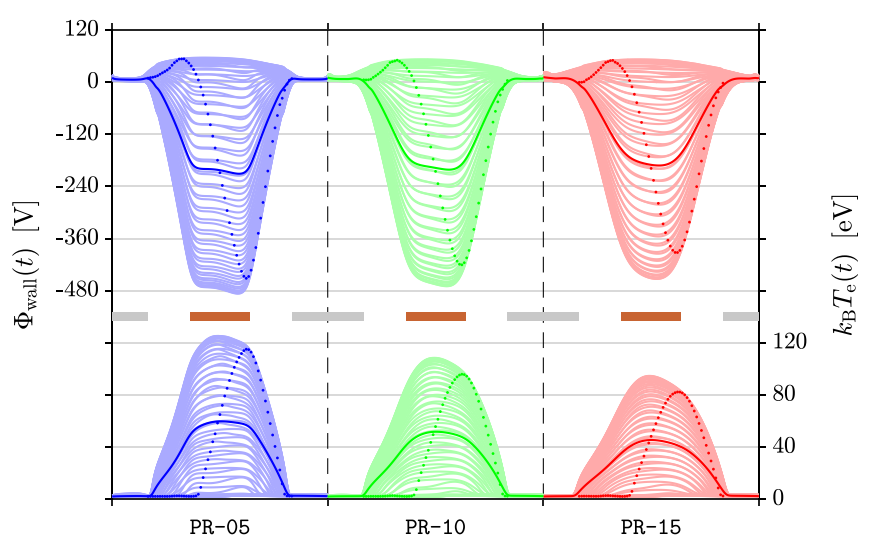

FIG. 13. Spatially and temporally resolved electron energy $k_{\mathrm{B}} T_{\mathrm{e}}(t)$ and electric potential $\Phi_{\text {wall }}(t)$ profiles along the surface of the discharge chamber wall (top) profiles near the surface discharge chamber wall (bottom) in PR05 (blue lines), PR-10 (green lines), and PR-15 (red lines). Solid lines denote the mean, while the dotted lines illustrate the oscillation of the respective profiles over one RF cycle. by the Macor discs from both the powered electrode and the grounded structure (see Fig. 1).

During $\tau_{-}$, the $\Phi_{\text {wall }}(t)$ axial profiles have a plateau shape where $\Phi_{\text {wall }}(t)$ is greatly negative in the region underneath the powered electrode. The electric potential at the sections of the discharge chamber wall underneath the grounded structure is close to zero but slightly positive $(6.7 \mathrm{~V}$ on average) due to the presence of the positive plasma potential. There is a steep gradient in $\Phi_{\text {wall }}(t)$ across the surface of the insulated section of the discharge chamber wall, suggesting that ions are drawn towards the negatively biased wall from a wider angle in the discharge chamber instead of only in the radial direction. During $\tau_{+}$, the powered sheath is collapsed, and $\Phi_{\text {wall }}(t)$ on the ungrounded sections of the discharge chamber wall becomes positive due to the positive $\Phi_{\mathrm{p}}(t)$ of the plasma bulk. The positive peak of $\Phi_{\text {wall }}^{+}$is also much flatter than the negative peak $\Phi_{\text {wall }}^{-}$or the mean $\Phi_{\text {wall }}$, which results in less ambiguity in the definition of $V_{\text {bias }}^{\prime}(8)$.

The emission region for secondary electrons is not limited to the section of the discharge chamber wall underneath powered electrode but extends to cover the insulated section as well. The $k_{\mathrm{B}} T_{\mathrm{e}}(t)$ axial profiles are low and flat in the emission region during $\tau_{+}$and likewise along the grounded section of the discharge cavity wall but for the whole RF cycle, indicating the absence of high energy secondary electrons in the respective regions during these periods. The $k_{\mathrm{B}} T_{\mathrm{e}}(t)$ axial profile only rises during $\tau_{+}$, as illustrated by the dotted line in each profile.

Figure 14 shows a $2 \mathrm{D}$ axisymmetric colour map of $k_{\mathrm{B}} T_{\mathrm{e}}$ in the discharge chamber at $3 \pi / 2 \mathrm{rad}$ (time-step 45 out of 60 ) of the RF cycle during the negative peak of $\Phi_{\text {wall }}(t)$ for PR05 (top), PR-10 (middle), and PR-15 (bottom). The colour scale ranges from $0 \mathrm{eV}$ (blue) to $127 \mathrm{eV}$ (magenta), with isocurves denoting every $20 \mathrm{eV} . k_{\mathrm{B}} T_{\mathrm{e}}$ peaks at $127 \mathrm{eV}, 111 \mathrm{eV}$, and $99 \mathrm{eV}$ for $\mathrm{PR}-05, \mathrm{PR}-10$, and $\mathrm{PR}-15$, respectively. The high energy secondary electrons indicate the full extent of the powered sheath during the negative peak of $\Phi_{\text {wall }}(t)$. CFD-ACE + is capable of producing plots like these for any tracked parameter with high spatial and temporal resolution (determined by the set mesh density and time-step size),
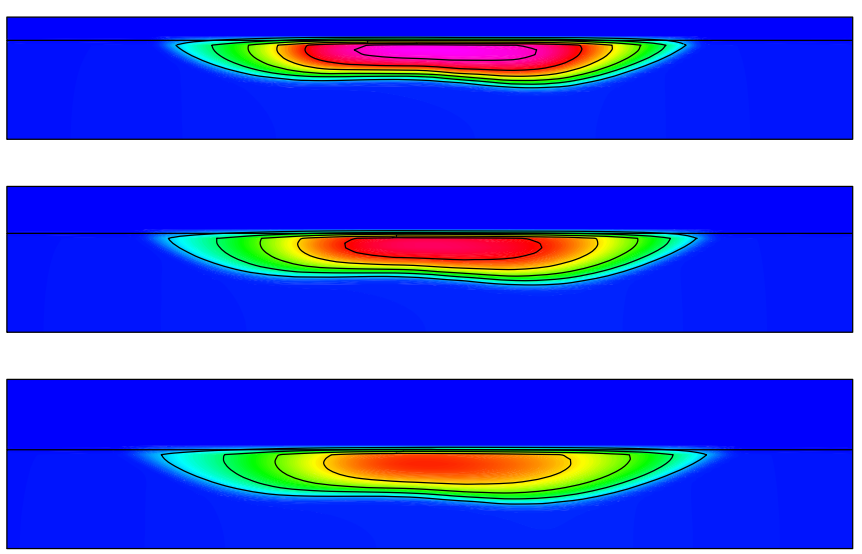

FIG. 14. 2D axisymmetric colour map of $k_{\mathrm{B}} T_{\mathrm{e}}$ in the discharge chamber at $3 \pi / 2 \mathrm{rad}$ (time-step 45 out of 60 ) of the RF cycle during the negative peak of $\Phi_{\text {wall }}(t)$ for PR- 05 (top), PR-10 (middle), and PR-15 (bottom). The colour scale ranges from $0 \mathrm{eV}$ (blue) to $127 \mathrm{eV}$ (magenta), with isocurves denoting every $20 \mathrm{eV}$. 
which may be viewed as an animation to demonstrate the temporal evolution of fluid, electrical, and plasma properties in the simulation domain.

\section{CONCLUSION}

PR is designed with a large geometrical area asymmetry of the grounded and powered electrodes in order to create a strong self-bias in the dielectric discharge chamber wall. The persistent negative DC bias sets up a steep potential drop across the powered sheath during the negative period of the RF cycle, which accelerates ions from the plasma bulk into the surface of the discharge chamber wall at very high velocities. Ion bombardment on the discharge chamber wall results in the emission of high energy secondary electrons, which are the primary source of ionisation in the discharge chamber, driving the strong central gamma mode peak in the plasma density. While this cause-and-effect sequence of events is understood in principle, it has not been experimentally quantified since access is limited by the restrictive geometry of the discharge chamber.

A comprehensive CFD-plasma simulation technique is employed to fully model the spatiotemporal behaviour of the PR discharge. First, the computed ion transit time across the powered sheath is on the order of a RF period $\left(\tau_{\mathrm{i}} \sim 1.40\right.$ $\tau_{\mathrm{RF}}$ ), demonstrating that the quasi-steady-state sheath circuit model for the $\tau_{\mathrm{i}} \gg \tau_{\mathrm{RF}}$ regime is no longer valid. Second, the CFD-plasma model shows that a portion of the RMS RF voltage applied to the powered electrode is dropped across the dielectric discharge chamber wall due to its nonzero capacitive impedance, resulting in a lower RF voltage amplitude on the plasma. As such, the definition of $V_{\text {bias }}$ (4) in the $\tau_{\mathrm{i}} \ll \tau_{\mathrm{RF}}$ regime, which assumes a plasma that is in direct electrically conductive contact with the powered electrode and exposed to the full applied RF voltage, becomes inaccurate. Third, the CFD-plasma model confirms the experimental observations that the RF voltage waveform responds asymmetrically to extraneous impedances in the RF circuit, whereby only the negative peak, corresponding to the maximum potential drop at the powered sheath and the high energy peak of the IED, is sensitive to changes in impedance. Thus, the traditional definition for the self-bias voltage as $V_{\text {bias }}^{\prime \prime}=\Phi_{\text {wall }}$ is also no longer valid.

Hence, an alternative definition of $V_{\text {bias }}^{\prime}(8)$ is suggested for the class of shielded plasma systems operating in the $\tau_{\mathrm{i}} \leqslant \tau_{\mathrm{RF}}$ regime. Using $\Phi_{\text {wall }}^{+}$in the case of PR, or in general the maxima envelope of the RF waveform on the plasmafacing surface of the shielding component, provides a more stable baseline for a more rigorous definition of the selfbias voltage, since it is characteristic of the plasma parameters and the geometry of the plasma system. Additional advantages of this definition are that it is applicable to nonsinusoidal waveforms and directly correlated with the measurable low energy peak of the IED in the powered sheath. $V_{\text {bias }}^{\prime}$ calculated from (8) may also be compared with $V_{\text {bias }}$ from (4) to quantify any deviances of the plasma system from the ideal case.

The definitive characterisation of the self-bias in PR is achieved by means of CFD-plasma simulations of three configurations of PR in which the discharge chamber wall thickness is varied. The simulation technique and numerical method are extensively documented and accurately reflect the experimental apparatus. The CFD-plasma model of PR has previously been verified against experimental measurements of power, plasma density, and spatiotemporal heating trends. Presently, the simulated self-bias behaviour in PR is demonstrated to be in very good agreement with theoretical calculations of parameters such as capacitance, impedance, charge, current, voltage, and power. The high degree of spatiotemporal resolution, details, and accuracy of the results impart confidence in the CFD-plasma model of PR. This simulation technique may be adapted to study other similar discharges in a comprehensive manner that is not possible with RF electrical circuit models or invasive experiments.

${ }^{1}$ E. A. Edelberg and E. S. Aydil, J. Appl. Phys. 86, 4799 (1999).

${ }^{2}$ C. Steinbrüchel, Appl. Phys. Lett. 55, 1960 (1989).

${ }^{3}$ H. Jansen, H. Gardeniers, M. de Boer, M. Elwenspoek, and J. Fluitman, J. Micromech. Microeng. 6, 14 (1996).

${ }^{4}$ D. M. Goebel and I. Katz, Fundamentals of Electric Propulsion, Ion and Hall Thrusters (Wiley, Hoboken, 2008).

${ }^{5}$ R. W. Boswell, "Plasma micro-thruster," WO2012151639A1, IFI CLAIMS Patent Services (15 Nov 2012).

${ }^{6}$ R. W. Boswell, C. Charles, P. Alexander, J. Dedrick, and K. Takahashi, IEEE Trans. Plasma Sci. 39, 2512 (2011).

${ }^{7}$ C. Charles and R. W. Boswell, Plasma Sources Sci. Technol. 21, 022002 (2012).

${ }^{8}$ T. A. Lafleur, P. Chabert, and J.-P. Booth, J. Phys. D: Appl. Phys. 46, 135201 (2013).

${ }^{9}$ B. G. Heil, U. Czarnetzki, R. P. Brinkmann, and T. Mussenbrock, J. Phys. D: Appl. Phys. 41, 165202 (2008).

${ }^{10}$ T. A. Lafleur, Plasma Sources Sci. Technol. 25, 013001 (2015).

${ }^{11}$ M. Wiebold, Y.-T. Sung, and J. E. Scharer, Phys. Plasmas 19, 053503 (2012).

${ }^{12}$ H. S. Butler and G. S. Kino, Phys. Fluids 6, 1346 (1963).

${ }^{13}$ K. Köhler, J. W. Coburn, D. E. Horne, E. Kay, and J. H. Keller, J. Appl. Phys. 57, 59 (1985).

${ }^{14}$ M. M. Salem and J. F. Loiseau, J. Phys. D: Appl. Phys. 29, 1181 (1996).

${ }^{15}$ J.-P. Booth, G. Cunge, N. Sadeghi, and R. W. Boswell, J. Appl. Phys. 82, 552 (1997).

${ }^{16}$ C. Charles, R. W. Boswell, and M. A. Lieberman, Phys. Plasmas 10, 891 (2003).

${ }^{17}$ H. B. Smith, "Computational studies of an asymmetric RF plasma using particle-in-cell techniques," Ph.D. thesis (The Australian National University, Canberra, 1994).

${ }^{18}$ H. B. Smith, C. Charles, R. W. Boswell, and H. Kuwahara, J. Appl. Phys. 82, 561 (1997).

${ }^{19}$ T. A. Lafleur and R. W. Boswell, Phys. Plasmas 19, 023508 (2012).

${ }^{20}$ M. A. Lieberman and A. J. Lichtenberg, Principles of Plasma Discharges and Materials Processing, 2nd ed. (Wiley, 2005).

${ }^{21}$ M. A. Lieberman, IEEE Trans. Plasma Sci. 16, 638 (1988).

${ }^{22}$ M. A. Lieberman, IEEE Trans. Plasma Sci. 17, 338 (1989).

${ }^{23}$ P. M. Vallinga, P. M. Meijer, and F. J. de Hoog, J. Phys. D: Appl. Phys. 22, 1650 (1989).

${ }^{24}$ V. A. Godyak and N. Sternberg, Phys. Rev. A 42, 2299 (1990).

${ }^{25}$ E. Kawamura, V. Vahedi, M. A. Lieberman, and C. K. Birdsall, Plasma Sources Sci. Technol. 8, R45 (1999).

${ }^{26}$ A. Metze, D. W. Ernie, and H. J. Oskam, J. Appl. Phys. 60, 3081 (1986).

${ }^{27}$ P. M. Vallinga and F. J. de Hoog, J. Phys. D: Appl. Phys. 22, 925 (1989).

${ }^{28}$ P. A. Miller and M. E. Riley, J. Appl. Phys. 82, 3689 (1997).

${ }^{29}$ M. A. Sobolewski, J. Appl. Phys. 95, 4593 (2004).

${ }^{30}$ C. Charles, A. W. Degeling, T. E. Sheridan, J. H. Harris, M. A. Lieberman, and R. W. Boswell, Phys. Plasmas 7, 5232 (2000).

${ }^{31}$ T. S. Ho, C. Charles, and R. W. Boswell, Phys. Plasmas 24, 084501 (2017).

${ }^{32}$ M. A. Sobolewski, Phys. Rev. E 62, 8540 (2000).

${ }^{33}$ M. A. Sobolewski, J. Appl. Phys. 90, 2660 (2001).

${ }^{34}$ T. S. Ho, C. Charles, and R. W. Boswell, Front. Phys. 4, 55 (2017). 
${ }^{35}$ A. Greig, C. Charles, and R. W. Boswell, IEEE Trans. Plasma Sci. 42, 2728 (2014).

${ }^{36}$ A. Greig, C. Charles, R. Hawkins, and R. W. Boswell, Appl. Phys. Lett. 103, 074101 (2013).

${ }^{37}$ A. Greig, C. Charles, N. Paulin, and R. W. Boswell, Appl. Phys. Lett. 105, 054102 (2014).

${ }^{38}$ A. Greig, C. Charles, and R. W. Boswell, Front. Phys. 3, 84 (2015).

${ }^{39}$ A. Greig, C. Charles, and R. W. Boswell, Phys. Plasmas 23, 013508 (2016).

${ }^{40}$ C. Charles, R. Hawkins, and R. W. Boswell, Appl. Phys. Lett. 106, 093502 (2015).

${ }^{41}$ A. Greig, C. Charles, and R. W. Boswell, Front. Phys. 2, 80 (2015).
${ }^{42}$ L. L. Alves, J. Phys.: Conf. Ser. 565, 012007 (2014).

${ }^{43}$ J. T. Guðmundsson and E. G. Thorsteinsson, Plasma Sources Sci. Technol. 16, 399 (2007).

${ }^{44}$ A. Greig, "Pocket rocket: An electrothermal plasma micro-thruster," Ph.D. thesis (The Australian National University, Canberra, 2015).

${ }^{45}$ C. Charles, A. Bish, R. W. Boswell, J. Dedrick, A. Greig, R. Hawkins, and T. S. Ho, Plasma Chem. Plasma Process. 36, 29 (2016).

${ }^{46}$ V. I. Kolobov, R. R. Arslanbekov, E. A. Bogdanov, S. Eliseev, and A. A. Kudryavtsev, International Conference on Phenomena in Ionized Gases (Granada, 2013).

${ }^{47}$ M. A. Sobolewski, J. Appl. Phys. 100, 063310 (2006). 